\title{
Sources of machine-induced background in the ATLAS and CMS detectors at the CERN Large Hadron Collider
}

\author{
R. Bruce $^{\mathrm{a}, *}$, R.W. Assmann ${ }^{\mathrm{b}, 1}$, V. Boccone ${ }^{\mathrm{a}}$, G. Bregliozzi ${ }^{\mathrm{a}}, \mathrm{H}$. Burkhardt ${ }^{\mathrm{a}}$, F. Cerutti $^{\mathrm{a}}$, \\ A. Ferrari ${ }^{a}$, M. Huhtinen ${ }^{a}$, A. Lechner ${ }^{a}$, Y. Levinsen ${ }^{a}$, A. Mereghetti ${ }^{a}$, N.V. Mokhov ${ }^{c}$, \\ I.S. Tropin ${ }^{\mathrm{c}}$, V. Vlachoudis ${ }^{\mathrm{a}}$ \\ ${ }^{a}$ CERN, Geneva, Switzerland \\ ${ }^{\mathrm{b}}$ DESY, Hamburg, Germany

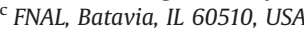

\section{A R T I C L E I N F O}

\section{Article history:}

Received 29 July 2013

Received in revised form

12 August 2013

Accepted 17 August 2013

Available online 29 August 2013

Keywords:

Circular colliders

Synchrotrons

Experimental backgrounds

Collimation

Large Hadron Collider

\begin{abstract}
A B S T R A C T
One source of experimental background in the CERN Large Hadron Collider (LHC) is particles entering the detectors from the machine. These particles are created in cascades, caused by upstream interactions of beam protons with residual gas molecules or collimators. We estimate the losses on the collimators with SixTrack and simulate the showers with FLUKA and MARS to obtain the flux and distribution of particles entering the ATLAS and CMS detectors. We consider some machine configurations used in the first LHC run, with focus on $3.5 \mathrm{TeV}$ operation as in 2011. Results from FLUKA and MARS are compared and a very good agreement is found. An analysis of logged LHC data provides, for different processes, absolute beam loss rates, which are used together with further simulations of vacuum conditions to normalize the results to rates of particles entering the detectors. We assess the relative importance of background from elastic and inelastic beam-gas interactions, and the leakage out of the LHC collimation system, and show that beam-gas interactions are the dominating source of machine-induced background for the studied machine scenarios. Our results serve as a starting point for the experiments to perform further simulations in order to estimate the resulting signals in the detectors.
\end{abstract}

(c) 2013 Elsevier B.V. All rights reserved.

\section{Introduction}

The Large Hadron Collider (LHC) [1,2] at CERN collides protons and heavy ions at unprecedented energies. The proton operation started at a beam energy of $3.5 \mathrm{TeV}$ in 2010 , which was raised to $4 \mathrm{TeV}$ in 2012 , while the aim is to achieve $7 \mathrm{TeV}$ in the future.

To ensure optimal performance of the experimental detectors, it is important to understand the background, which can come from several sources. The most important is particles originating from the cascades caused by the proton-proton collisions themselves [3]. Another source of background, believed to be the second most important, is particles entering the detector from the accelerator. This is the main focus of our paper. These particles are produced in the hadronic and electromagnetic showers resulting from beam protons interacting either with upstream collimators-because this background is caused by the proton halo we refer to it as beam-halo-or residual gas molecules inside the vacuum pipe. We call this latter source beam-gas. Background can also originate from a cross-talk between different experiments, i.e.

\footnotetext{
* Corresponding author. Tel.: +41227672539.

E-mail address: roderik.bruce@cern.ch (R. Bruce).

${ }^{1}$ On leave from CERN, Geneva, Switzerland.
}

particles scattered in a collision travel to another experiment where they cause a shower. This source is not treated in detail here as it is likely to give smaller contributions-some general remarks are made in Section 9.

Machine-induced background depends strongly on the machine configuration, e.g. on the beam intensity and energy, the residual gas densities in the vacuum chamber, the collimator settings, and the machine optics. Previous studies for the nominal $7 \mathrm{TeV}$ machine, performed before the startup of the LHC, can be found in Refs. [3-11]. In this paper, we consider instead the actual proton beam and vacuum conditions observed in the LHC, with the focus on 2011 but with some results also for the 2010 run. We describe simulations of beam-halo and beam-gas for the two high-luminosity general-purpose experiments: ATLAS [12] and CMS [13]. We compare simulation results between different Monte Carlo codes and normalize the results with our best estimate of the machine conditions during a typical fill in 2011.

First we give a general overview of the LHC machine and collimation system in Section 2 followed by a description of the used simulation tools in Section 3. The assumed beam and vacuum conditions are presented in Section 4 followed by descriptions, in Sections 5-7, of the simulation setup and results for the different components of the background. Finally, in Section 8 we compare 
the magnitudes of the rates of particles entering the detector from beam-halo and beam-gas. We use logged beam intensity and beam loss data in order to normalize the results.

Our simulations stop at an interface plane between the machine and the detector, which is defined to be at $22.6 \mathrm{~m}$ upstream of the collision point. Our final output, distributions of particles crossing the interface plane, serves as a source term for further simulations of the resulting signals in the experimental detectors.

\section{LHC machine and collimation}

As shown in Fig. 1, the LHC consists of 8 arcs and 8 straight sections, called insertion regions (IRs) with different functionality. Four of them house interaction points (IPs) where the two counter-rotating beams, called B1 and B2, collide inside the LHC experiments. The beams are injected in IR2 and IR8 and extracted in IR6. The general machine parameters of the 2010 and 2011 runs, including the most important collimator settings, are summarized in Table 1. The major differences between the years are the higher beam intensity and luminosity in 2011.

Beam losses outside the experiments are unavoidable during collider operation. The beam halo is continuously repopulated and has to be cleaned by the collimation system $[1,14-16]$, so that the losses in the cold superconducting magnets are kept safely below the quench limit. The collimation system is mainly located in two dedicated insertions (IR3 for momentum cleaning and IR7 for betatron cleaning, see Fig. 1). Most collimators consist of two movable jaws, with the beam passing in the center between them.

The collimation system is composed of several stages, with the primary collimators, called TCP, closest to the beam, followed by secondary collimators (TCS) and absorbers. For optimal performance, the halo particles should first hit a TCP, and the TCS and absorbers should only catch the losses that are scattered out of other upstream collimators. Furthermore, special dump protection collimators are installed in IR6 at the beam extraction [1]. Tertiary collimators (TCTs) made of tungsten are installed in the experimental IRs about $150 \mathrm{~m}$ upstream of the collision points, in order to provide local protection of the quadrupole triplets in the final



Fig. 1. The schematic layout of the LHC (the separation of the two rings is not to scale) shown in a global coordinate system with the origin in the ATLAS collision point. The two beams collide at the four experiments ATLAS, ALICE, CMS and LHCb.
Table 1

Typical proton running conditions in the LHC in 2010 and 2011. Here $\beta^{*}$ refers to the optical $\beta$-function at the collision point, and the collimator settings are shown in units of beam standard deviations $\sigma$, calculated assuming a normalized beam emittance of $3.5 \mu \mathrm{m}$.

\begin{tabular}{lcc}
\hline Parameter & 2010 & 2011 \\
\hline Beam energy $(\mathrm{TeV})$ & 3.5 & 3.5 \\
Bunch intensity $\left(10^{11} \mathrm{p}\right)$ & 1.2 & 1.5 \\
Number of bunches & 368 & $\leq 1380$ \\
$\beta^{*}$ in IR1, IR5 $(\mathrm{m})$ & 3.5 & $1.0-1.5$ \\
crossing angle in IR1, IR5 $(\mu \mathrm{rad})$ & 200 & 240 \\
Peak luminosity $\left(10^{32} \mathrm{~cm}^{-2} \mathrm{~s}^{-1}\right)$ & 2 & $\leq 40$ \\
TCP cut IR7 $(\sigma)$ & 5.7 & 5.7 \\
TCS cut IR7 $(\sigma)$ & 8.5 & 8.5 \\
TCP cut IR3 $(\sigma)$ & 12.0 & 12.0 \\
TCS cut IR3 $(\sigma)$ & 15.6 & 15.6 \\
TCT cut $(\sigma)$ & 15.0 & 11.8 \\
\hline
\end{tabular}

focusing system and to decrease experimental background. In front of each experiment, there is one TCT in the horizontal plane (TCTH) and one in the vertical plane (TCTV).

In spite of the sophisticated design and high efficiency, a small number of protons hitting the TCPs are not absorbed by the downstream cleaning system. Some of them are intercepted by TCTs. Elastic beam-gas interactions far from the detectors can also kick protons directly onto the TCTs without passing through other collimators. Parts of the high-energy showers induced by these losses can propagate into the detectors and cause background, even though the experiments are surrounded by a heavy shielding. This is true in particular for high-energy muons, for which the shielding is less efficient. They can cause large radiative energy deposits in calorimeters that could be erroneously reconstructed as jets. Therefore, we present the results for muons more in detail than for other particle species.

The effectiveness of the collimation hierarchy in protecting the aperture depends on the transverse openings of the collimators as well as on their longitudinal placement in terms of betatron phase advance [17]. In transverse phase space, a phase advance of an odd multiple of $\pi / 2$ is needed to convert an angular kick, given by for example a beam-gas scattering event, to a maximum spatial offset. In locations where the phase advance is a multiple of $\pi$, the scattering is again manifested as an angular offset. For scattering in a collimator, the dynamics is more complicated as the scattering angle is added to a particle which has already a large spatial offset [17]. The performance of the collimation system is usually measured in terms of the local cleaning inefficiency $\eta$, which is defined as the ratio of local losses per meter to the total losses on collimators.

\section{Simulation tools}

\subsection{SixTrack}

To estimate the distribution of protons impinging on the collimators and machine aperture close to the experiments but caused by initial interactions far away we use SixTrack [18], including a special routine for collimation $[19,20]$. The cases studied with SixTrack are beam-halo and distant beam-gas interactions, where scattered particles travel over large parts of the LHC ring or even make several turns before being lost. SixTrack is a multi-turn tracking code that takes into account the full six-dimensional phase space including synchrotron oscillations in a symplectic manner. SixTrack performs a thin-lens ${ }^{2}$ element-by-element tracking through the magnetic lattice.

\footnotetext{
${ }^{2}$ All magnets are approximated as zero-length elements and their strength are re-matched to reproduce the same beam optics as with thick elements.
} 
During the tracking, the particle coordinates are checked against a detailed aperture model with $10 \mathrm{~cm}$ longitudinal precision. If a particle hits the aperture, it is considered lost, except if this occurs in a collimator. In the latter case, a built-in Monte Carlo code [19] is used to simulate the particle-matter interaction, including the effects of multiple Coulomb scattering, ionization, single diffractive scattering, and point-like elastic and inelastic scattering. When an inelastic event occurs inside a collimator the particle is considered lost, otherwise the magnetic tracking continues. The simulation output contains coordinates of all losses. SixTrack, in combination with a second step of a FLUKA [21-23] simulation of the local showers, has been successfully benchmarked with experimental data of provoked losses in the LHC [24].

\subsection{FLUKA and MARS}

To simulate the showers close to the detectors, possibly starting from the output of a SixTrack simulation of nearby losses, we use FLUKA [21-23,25] and MARS [26,27]. Both codes are fully integrated particle physics Monte Carlo simulation packages, based on state-of-the-art models of particle interactions, which track initial particles and all created secondaries in the induced cascades through a user-defined geometry.

FLUKA and MARS are developed independently and we use separate implementations of the 3D geometries around the detectors. Using two completely independent simulations allows us to detect possible errors or inconsistencies and increases the confidence in the final results. It serves also as a demonstration of the consistency between the codes, which is of value for other applications too.

\subsection{VASCO}

In order to calculate the loss rate from beam-gas interactions, detailed maps of the gas densities and composition in the LHC are required. The measurements from pressure gauges are not sufficient, as local pressure bumps could appear in between them. Instead we use the VASCO $[28,29]$ code to simulate the pressure for the relevant beam conditions.

VASCO treats the vacuum system as a sequence of finite elements linked with boundary conditions that depend on the geometry of the beam pipe, surface materials, temperature, surface treatment, and conditioning along the accelerator ring. This results in a set of partial differential equations which are solved numerically. The simulation accounts not only for diffusion due to the pressure gradients and pumping but also for beam-induced dynamic effects such as electron and photon-stimulated outgassing due to synchrotron radiation and the pressure increase from electron cloud.

\section{Machine conditions}

In order to quantitatively estimate rates of particles entering the experiments and compare the different sources of background, the results are normalized by real conditions in the LHC. All LHC fills are numbered in chronological order and for our normalization we have selected fill 2028 on July 16, 2011. It was chosen as being representative for the 2011 operation in terms of experimental background [30]. The time evolution of the luminosity and the beam intensity are shown in Fig. 2. As can be seen, the loss rates from the two beams are not identical, coming from the fact that the non-collisional losses are different. This varies slightly from fill to fill and the asymmetries are confirmed by beam-loss measurements in the two beams over the year 2011 [31]. The physical reason for the differences between the beams is unknown.

The time with colliding beams was about $3 \mathrm{~h}$ in this fill. From the intensity data $N(t)$ in Fig. 2 we can extract the loss rate $N^{\prime}(t)$
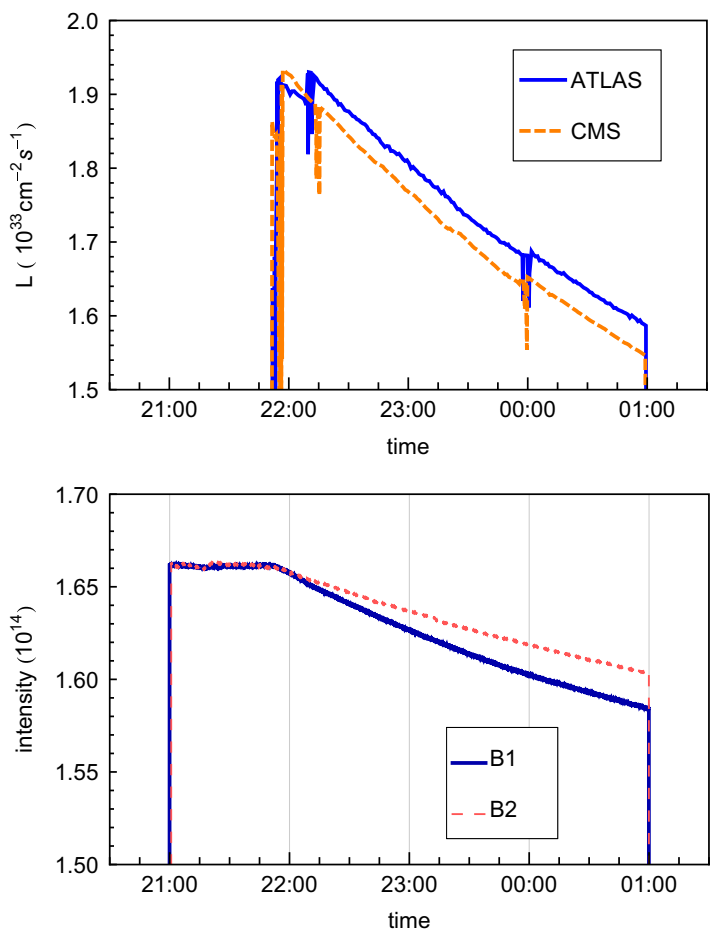

Fig. 2. The measured time evolution of luminosity $\mathcal{L}$ in ATLAS and CMS and intensity in both beams during fill 2028.

and then calculate the instantaneous lifetime $T_{l}$ of the beam as

$T_{l}(t) \approx N(t) / N^{\prime}(t)$.

From the data in Fig. 2, an initial intensity of $1.66 \times 10^{14}$ and an initial loss rate of about $1 \mathrm{GHz}$ are obtained, resulting in an initial lifetime of about $50 \mathrm{~h}$. This loss rate has several different contributions: collisional losses in the IPs, beam-gas scattering around the whole ring, and losses on the collimators of halo particles drifting out to large amplitudes. For the normalization of our simulations, we examine each of these contributions in detail.

The instantaneous beam loss rate $N_{\mathcal{L}}^{\prime}(t)$ of particles at an experiment with a luminosity $\mathcal{L}$ is given by

$N_{\mathcal{L}}^{\prime}(t)=\mathcal{L}(t) \sigma_{i}$

where $\sigma_{i}$ is the interaction cross-section between the colliding beam particles. For our purposes, we assume that most particles undergoing inelastic interactions in the collision points are lost locally (this is true except in the case of diffractive events); elastic interactions are instead more likely to send protons on the primary collimators. Even if this is not strictly true for all of them, we use this assumption to estimate an upper limit on the loss rate at the TCPs. In reality, the elastic interactions send protons also onto other collimators, such as the TCTs in other IRs. This corresponds to the additional contribution to the background referred to above as cross-talk (see also Section 9). Using an inelastic cross-section $\sigma_{i}=73.5 \mathrm{mb}$ [32], we obtain $N_{\mathcal{L}}^{\prime}=1.4 \times 10^{8} \mathrm{~Hz}$ at the beginning of the fill for each highluminosity experiment.

Simulations of the gas densities around the ring are done with VASCO. Separate simulations were performed for IR1, IR2, IR4, IR7, IR8, and the arc in B1. Because of the very similar layout and conditions, ${ }^{3}$ the pressure profile in IR5 was assumed to be the same as in IR1. Furthermore, the momentum collimation insertion IR3 was assumed to have the same average as the IR7 betatron

\footnotetext{
${ }^{3}$ The layout is the same for $|z|>19 \mathrm{~m}$ and we do not consider beam-gas interactions at $|z|<22.6 \mathrm{~m}$ in our study.
} 
cleaning insertion and we also use the IR4 average pressure in IR6. This approximation, although not strictly true, is justified by the fact that the few collimators in IR6 cause only local pressure spikes that do not significantly affect the average, which is the important quantity when studying resulting losses far away. B2 was assumed symmetric to B1, except in IR2 and IR8, where IR2 B2 was presumed equal to IR8 B1 and vice versa in order to account for the layout of the injection regions.

The simulated local gas densities in IR1 are shown in Fig. 3. The IP is located at $z=0$, and the two symmetric parts with higher pressure on each side at about $30 \mathrm{~m}$ from the IP correspond to the final focusing quadrupoles. Worth noting is also the asymmetric pressure peak at $z=-145 \mathrm{~m}$, corresponding to the TCTs of the incoming beam, and the lower pressure spike on the other side of the IP at a passive absorber for neutral particles (called TAN). Here $z$ denotes the longitudinal distance from the IP in a right-handed Cartesian system, where $x$ is directed towards the outside of the ring and $y$ points upwards.

Given the gas densities, decomposed in elemental constituents, and the revolution time $T_{\text {rev }}$, the local interaction probability $p_{j}$ of a proton on a gas nuclei of type $j$ per time and length units can be calculated as

$p_{j}=\frac{\sigma_{j}}{T_{\text {rev }}} \rho_{j}(s)$.

Here $\rho_{j}$ is the number of nuclei of species $j$ per volume and $\sigma_{j}$ is the cross-section for beam protons interacting it. Based on the gas densities in Fig. 3, $p$ in IR1, summed over $j$, is shown in Fig. 4.

The simulated gas densities assumed around the LHC ring are shown in Fig. 5, where we use a moving coordinate system with $s$ being the distance traveled along the ideal beam orbit around the ring. For better readability we set $s=0$ at the center of IR6. The

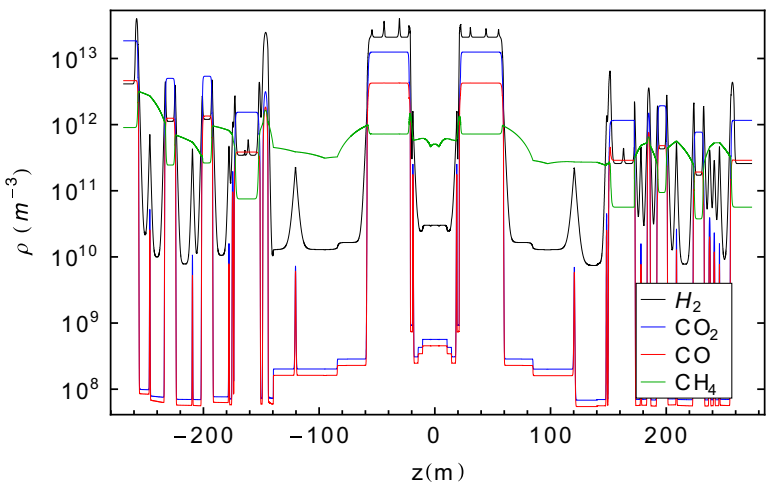

Fig. 3. The simulated density $\rho$ of different gas molecules from VASCO in IR1 during fill 2028. The IP is located at $z=0$ and the beam moves along positive $z$, i.e. from left to right in the figure.

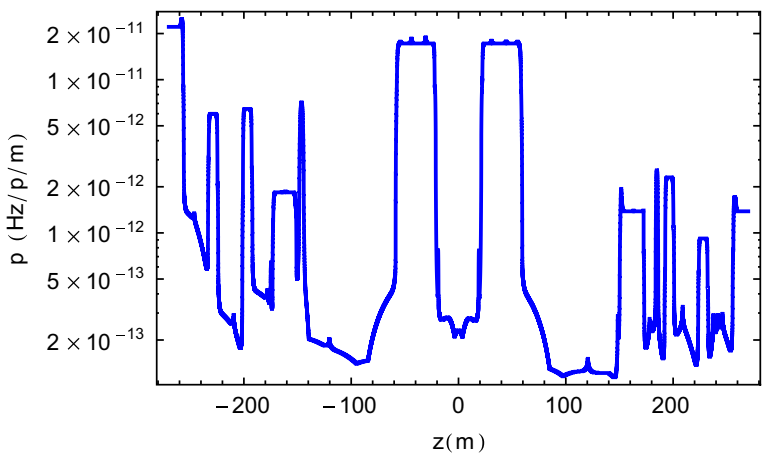

Fig. 4. The local beam-gas interaction probability $p$ in IR1 during fill 2028, summed over all gas species shown in Fig. 3. The beam moves along positive $z$, i.e. from left to right in the figure. molecular densities have been decomposed in atomic densities in order to easier assess the interactions with beam protons. The dominating gas species in the arcs is oxygen, which becomes even more pronounced when accounting for the interaction crosssections presented in Table 2.

Using the beam intensity $N$, the total rate of interactions $N_{\text {gas }, j}^{\prime}$ between beam protons and gas nuclei $j$ can be calculated by integrating the $p_{j}$ around the ring circumference $C$

$N_{\text {gas }, j}^{\prime}=N \int_{0}^{C} p_{j} \mathrm{~d} s$

The total resulting loss rate, summing Eq. (4) over inelastic and elastic interactions on all gas constituents, is about $1.3 \times 10^{8} \mathrm{~Hz}$, assuming an intensity of $1.66 \times 10^{14}$ as in the beginning of fill 2028 (see Fig. 2). Even though elastic beam-gas is likely to send protons on the primary collimators, we do not include it in the halo losses, since we simulate all beam-gas losses separately.

The loss rates and resulting beam lifetimes, calculated with Eq. (1), are summarized in Table 3, where the halo losses are assumed to be the remainder when losses from luminosity and beam-gas have been subtracted from the total.

These losses are mainly driven by the beam-beam effect, elastic scattering at the IPs, noise on RF and feedback systems, and intrabeam scattering. As can be seen, the halo losses are in this case about double the luminosity losses. The luminosity is quite similar between different fills, while the beam-halo losses can vary significantly. In some other fills, they contribute only about

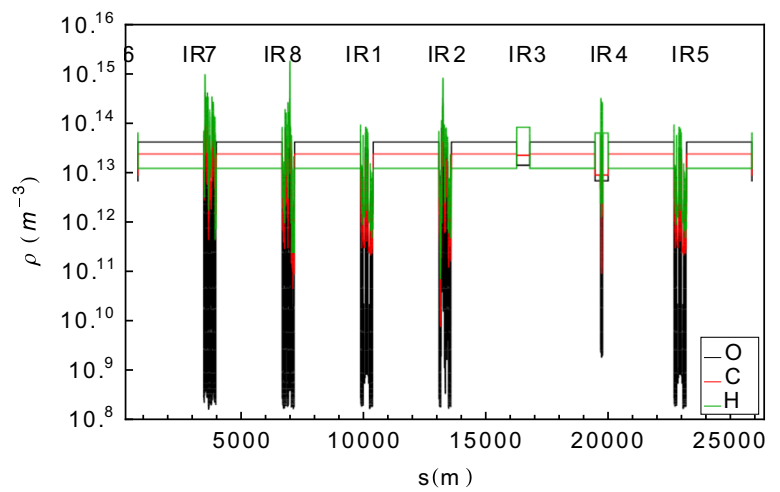

Fig. 5. The density $\rho$ of different gas atoms around the LHC ring as simulated with VASCO for fill 2028 in 2011. For better readability, the $s$-coordinate around the ring has been chosen to have $s=0$ at the center of IR6.

Table 2

Elastic and inelastic cross-sections between protons at $3.5 \mathrm{TeV}$ and different nuclei, extracted from FLUKA.

\begin{tabular}{lrrr}
\hline Cross-section type & H & C & O \\
\hline Elastic cross-section $(\mathrm{mb})$ & 8 & 107 & 139 \\
Inelastic cross-section $(\mathrm{mb})$ & 37 & 258 & 316 \\
\hline
\end{tabular}

Table 3

Beam loss rates and lifetimes from different processes at the beginning of fill 2028 as calculated from pressure distributions, interaction cross-sections and beam intensity data. The luminosity is summed over the four experiments, where the contributions from ATLAS and CMS make up 93\%.

\begin{tabular}{lrc}
\hline Process & Loss rate $(\mathrm{MHz})$ & Lifetime $(\mathrm{h})$ \\
\hline Luminosity (inelastic) & 300 & 154 \\
Inelastic beam-gas & 90 & 520 \\
Elastic beam-gas & 40 & 1225 \\
Halo on TCP & 570 & 81 \\
Total losses & 1000 & 46 \\
\hline
\end{tabular}


the same amount as luminosity to the total intensity decay. Beamgas interactions make up for about $13 \%$ of the total loss rate. These obtained loss rates are used later in order to normalize the simulations.

\section{Simulations of beam-halo}

To simulate the beam-halo induced showers entering the detectors, we use a two-step approach. First we simulate the cleaning by the LHC collimation system with SixTrack and record the coordinates of the inelastic interactions on the TCTs in front of the experiments. The second step is to use these coordinates as starting conditions for shower simulations with FLUKA and MARS.

\subsection{SixTrack simulations}

The SixTrack simulation of the beam cleaning in the LHC is performed using the methods outlined in Refs. [33,34]. We do not include diffusion, as it is insignificant over the short timescale considered. Instead we start the tracking with halo particles that have an amplitude high enough to hit the primary collimators. This approach significantly increases the efficiency of the simulation, since no computing time is lost tracking particles in the beam core that never hit any collimator.

Apart from the 2011 machine, we simulate also the 2010 configuration for comparison, using the machine parameters shown in Table 1. For each configuration, separate simulations are done for the two beams and for the horizontal and vertical planes, i.e. eight different simulations. As the collimators are more open in IR3 than in IR7 (see Table 1) and intercepts significantly less particles, ${ }^{4}$ and the measured leakage to the TCTs is about the same as from IR7, we simulate only the betatron losses in IR7. We track over 200 turns, which are sufficient for the vast majority of the initial particles to be lost and to study the multi-turn effects involving several scattering events in different collimators. For each studied case, we simulate $6.4 \times 10^{7}$ halo particles.

The simulated $\eta$ around the B1 ring is shown in Fig. 6 for 2011 conditions and the case of particles hitting first the horizontal TCP. The result is qualitatively similar to the other planes and machine configurations. As expected, the main loss location is in IR7, with the highest number of impacts on the TCPs and with decreasing impacts on downstream collimators.

The coordinates of inelastic interactions, as obtained from SixTrack, inside the TCTs in IR1 and IR5 are used as starting conditions for the shower simulations with FLUKA and MARS, described in Section 5.2. Example histograms showing the distributions of the depth of the first inelastic interactions in the TCTHs for the 2011 simulation are shown in Fig. 7. As can be seen, the impact distribution has quite significant variations between different IRs and beams. These variations depend on if any other TCTs or collimators were passed on the way from IR7 as well as on differences in betatron phase advance from the IR7 collimators. Similar variations were seen in the 2010 simulation.

Fig. 6 shows the simulated losses with a perfect collimation system. Including imperfections of the collimators ${ }^{5}$ increases the cleaning inefficiency and the TCTs see on average about 5 times higher losses. The leakage to the TCTs, defined as the ratio of losses

\footnotetext{
${ }^{4}$ This observation holds true especially for the first part of the fills. Towards the end of fills, when the momentum tails are more populated, losses have in some cases been observed in IR3 at a level comparable to IR7. Depending on fill, one might thus have to simulate in addition the cleaning in IR3 if the end of the fill is considered.

${ }^{5}$ We deploy random misalignments around the beam orbit, random tilt errors, random errors of the gap opening, and a non-flat surface, using the parameters defined in Ref. [34].
}



Fig. 6. LHC cleaning inefficiency $\eta$ in fill 2028, in cold and warm elements and collimators, as simulated with SixTrack. The horizontal TCP in IR7, beam 1, was hit first by the halo, and has an inefficiency slightly above 1 , since the losses happen over a length of $60 \sim \mathrm{cm}$.

at the TCTs to the total losses around the ring, is compared to measurements in Ref. [24]. It is found that, when the showering from the collimators to the adjacent beam loss monitors is included, a good agreement is achieved. The average leakage, including imperfections, is summarized in Table 4 for the 2011 machine. The observed leakage is significantly higher to IR1 than to IR5, which is explained by the differences in the phase advance conditions from IR7. The asymmetries are very well reproduced by measurements [24].

\subsection{Shower simulations of beam-halo}

IR1 and IR5 are very similar in terms of layout of the beam-line elements and the resulting beam envelope, except that in IR5 both the crossing angle and the envelopes are rotated by $90^{\circ}$ with respect to IR1. After the straight section, the layout is identical. Other differences between the IR1 and IR5 geometries are present at larger radial distance from the beam line in terms of support tunnels.

In FLUKA, the IR1 geometry has been implemented out to $550 \mathrm{~m}$ upstream of the IP, while in IR5, only the section up to the TCTs at $z=150 \mathrm{~m}$ was used. The extended geometry going out to $z=550 \mathrm{~m}$ is needed $[10,11]$ for the beam-gas simulations described in Section 7, while for beam-halo only the part between the TCTs and the interface plane is relevant. In MARS, the IR5 geometry was implemented independently to $z=550 \mathrm{~m}$, with the difference that the smaller support tunnels outside the main beam line are not included in the MARS model. As an example, the IR1 FLUKA geometry between the interface plane and the TCTs is shown in Fig. 8. The IR5 MARS geometry in this region is identical except for minor layout differences between the IRs. Both models contain a detailed material composition of all elements and are restricted to only one side of the IP (incoming B2). Because of the symmetry, the same geometries, after a coordinate transformation, were used to simulate the beam coming also from the other side. Some differences in the layout of the support tunnels exist at large radii (above $2 \mathrm{~m}$ ), which may introduce a small error in the B1 simulations. However, as all magnetic elements and optical functions are identical for the incoming beams, the introduced uncertainty only affects shower particles passing far from the beam line.

Both the FLUKA and MARS models include magnetic field maps in the final focusing quadrupoles, some other quadrupoles in the straight section, the main arc dipoles and quadrupoles, as well as in the first dipole after the IP, called D1. Between the D1 dipoles on either side of the IP, the beams are superimposed so that, if no 

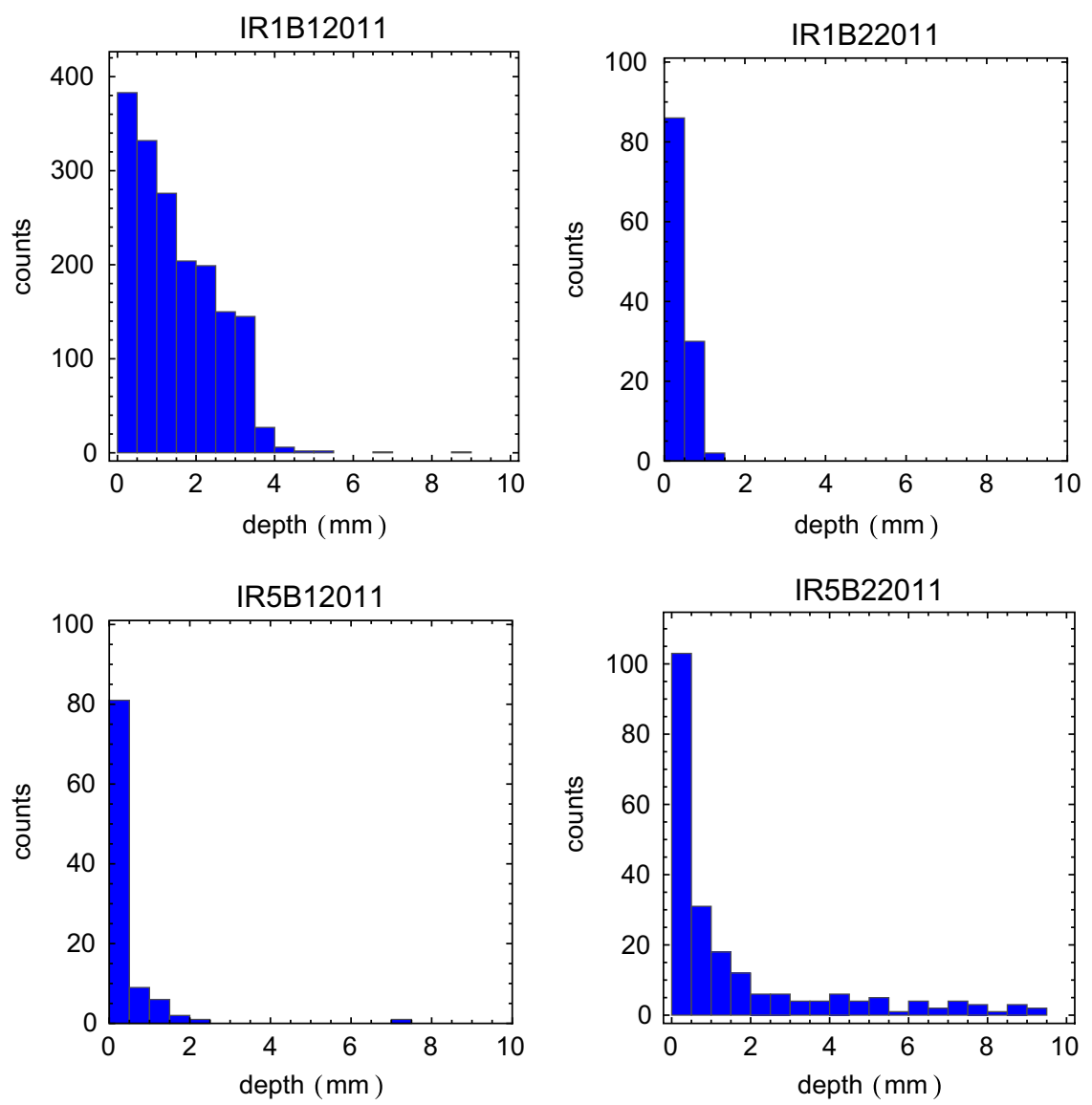

Fig. 7. Simulated positions of inelastic interactions from SixTrack on the TCTH in different IRs in the 2011 machine.

crossing angle were present, they would follow the same trajectory through the $\mathrm{IP}^{6}$ as shown in Fig. 8. The field maps are sampled on a grid with a maximum $5 \mathrm{~mm}$ spacing and interpolated linearly at runtime. As an example, a transverse crosssection of the FLUKA model of the first quadrupole of the inner triplet, called Q1, with the magnetic field superimposed, is shown in Fig. 9. In some other magnets (called MQY and D2), an analytic field is used inside the beam pipe only in FLUKA, while the corresponding field maps are still used in MARS. The correctors are not powered, meaning that there is no crossing angle. A study of the possible influence of the crossing angle is left as future work.

A major difference between the simulations with FLUKA and MARS is that we run FLUKA in an analogue mode, meaning that all created particles are tracked, while a sophisticated biasing was used in MARS. The main purpose of the MARS biasing in this application was to enforce production of rare particles, such as prompt and Bethe-Heitler muons and photo-hadrons, at the price of introducing fluctuating statistical weights and losing correlations. The analogue simulations have the advantage that complete events are stored, so that all correlations between particles are kept. On the other hand, the biasing results in a significant decrease of the required computing time. In both FLUKA and MARS, energy cutoffs at $20 \mathrm{MeV}$ were used in this study, motivated by the fact that particles with lower energy are blocked by the detector shielding. A set of FLUKA simulations were also done with higher cutoffs of $20 \mathrm{GeV}$ in order to increase the statistics for highenergy muons.

\footnotetext{
${ }^{6}$ At larger $z$, outside Fig. 8, the orbits are made parallel again, but with a separation, by another dipole (D2).
}

Table 4

Fraction of particles lost on the TCTs to the total losses initially impinging on the IR7 primary collimators, as simulated with SixTrack for the 2011 machine, per IR and beam. We show the sum of losses on horizontal and vertical TCTs, averaged over the simulations with initial hits on the horizontal and vertical primary collimators. The shown results are the average values when random imperfections of the collimators are included.

\begin{tabular}{lll}
\hline Leakage fraction & B1 & B2 \\
\hline IR1 & $1.0 \times 10^{-4}$ & $1.3 \times 10^{-4}$ \\
IR5 & $5.2 \times 10^{-5}$ & $1.6 \times 10^{-5}$ \\
\hline
\end{tabular}

The coordinates of inelastic interactions (including diffractive events) from SixTrack, shown in Fig. 7, were used as starting conditions for the shower simulations. Thus, the total crosssection for inelastic events in the TCTs is taken from SixTrack, while the fraction between different inelastic event types, and the respective exclusive particle production, is simulated by FLUKA or MARS. It should be noted, however, that up to the impact on the TCTs, including possible previous hits on other collimators, all physics modeling is handled by SixTrack.

An example of the source term, superimposed on the TCT geometry in FLUKA for B1 coming into IR1, is shown in Fig. 10 for the 2011 machine. About $4.8 \times 10^{6}$ primary events were launched in FLUKA for each case.

The simulated energy and radial distributions at the interface plane, for IR5 B2 in 2010 and from both FLUKA and MARS, are presented in Fig. 11. The dominating particle types are photons, electrons and positrons at low energy, muons in the mid-range ( $10 \mathrm{GeV}$ to a few hundred $\mathrm{GeV}$ ), and protons at energies close to the initial beam energy. Neutrons give significant contributions at all energies. Most of the total energy is carried by the protons, 


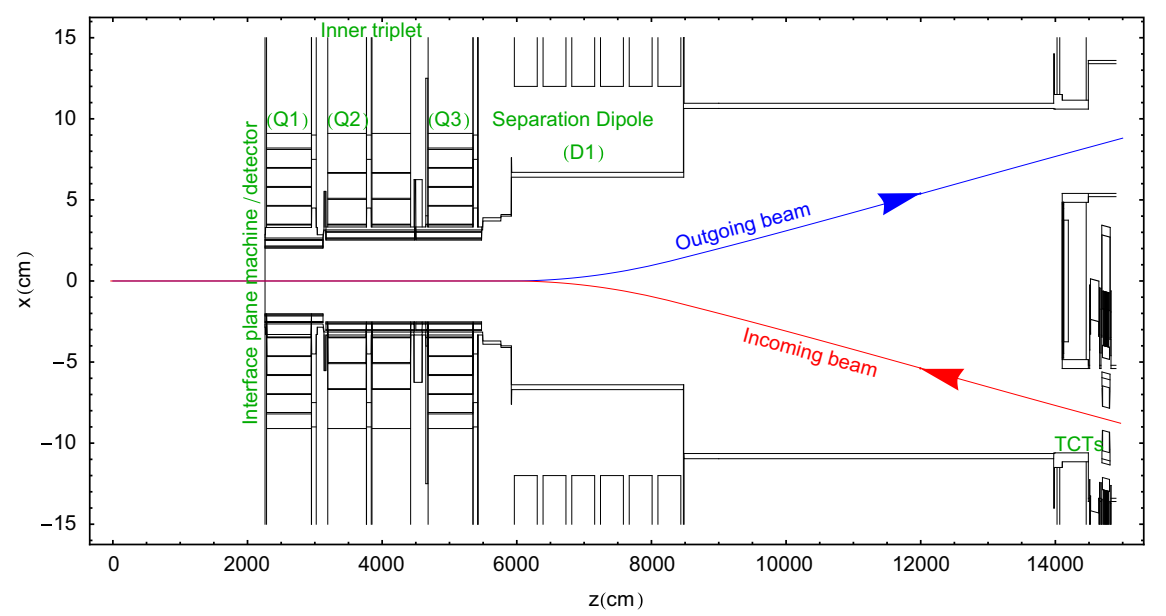

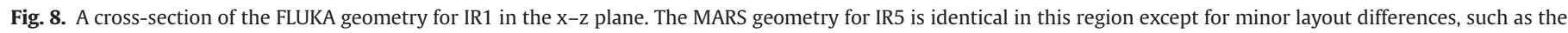
beam screen orientation. The interface plane to the experiment, where the model stops, is seen at $22.6 \sim \mathrm{m}$ as well as the incoming B2 and the outgoing B1.



Fig. 9. The transverse cross-section of the first quadrupole in the FLUKA geometry for IR1 with the magnetic field map superimposed.

as can be seen in the azimuthal energy distribution in Fig. 14. For protons, the energy distribution has a peak close to the $3.5 \mathrm{TeV}$ beam energy. It consists of protons suffering only a minor energy loss, for example through single diffractive events. These highenergy protons are all at very small radii. The total energy reaching the interface plane, per $3.5 \mathrm{TeV}$ proton hitting the TCTs, is on the order of $1 \mathrm{GeV}$.

Radially, most of the particles are found in the beam pipe. The distribution is approximately flat in the air extending to $r=1 \mathrm{~m}$ around the beam pipe and then falls off rapidly in the shielding between $r=1 \mathrm{~m}$ and $r=3 \mathrm{~m}$. It can also be seen that the most important particles at larger radii are muons.

It should be noted that an excellent agreement is found between the energy distributions from FLUKA and MARS, in spite of both the physical models and the geometry being implemented independently. Even though the energy distributions span over more than 8 orders of magnitude, the ratio stays in most cases within a factor 3 . Radially, the agreement is again very good except at $r>200 \mathrm{~cm}$. However, it should be noted that the support tunnels, not implemented in the MARS model, provide free air paths for particles in FLUKA. Thus, the FLUKA simulation shows

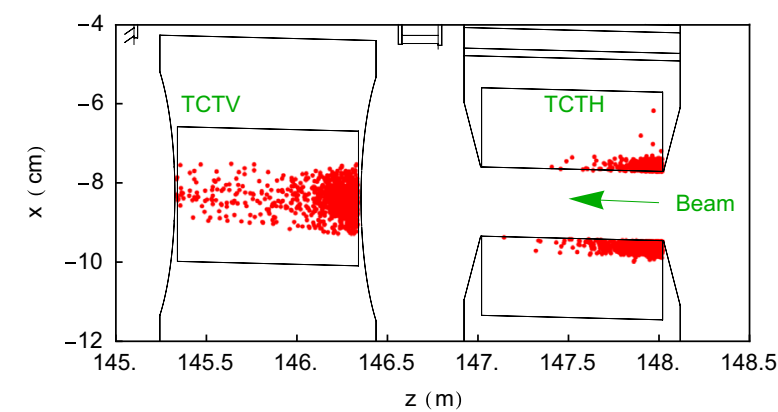

Fig. 10. Simulated positions of inelastic interactions from SixTrack on the horizontal and vertical TCTs, spanning over all vertical coordinates and superimposed on a slice of the FLUKA geometry in the horizontal plane for $y>0$ so that the TCTV is visible. The case shown is in IR1 B1 for the 2011 machine, mirrored to positive $z$. The rectangles enclosing the positions of the interactions are the tungsten inserts of the collimators.

higher fluences, in particular for muons. With this in mind we consider the agreement as very good, also in view of the high complexity of the simulation and the many orders of magnitude spanned by the distributions. At $r>600 \mathrm{~cm}$ the statistical error becomes dominant. The agreement between the codes increases our confidence in the results.

Some simulated energy distributions at the interface plane from FLUKA, at both IRs, for both beams and for 2010 and 2011 conditions, are shown in Fig. 12. We have divided the results by the corresponding FLUKA results in Fig. 11 for an easier comparison. A value of one thus means that the distribution is identical to the result in Fig. 11. A similar comparison of the radial distribution of muons is shown in Fig. 13.

An important conclusion from Fig. 12 is that for all TCT starting conditions, the shapes of the energy distributions at the interface plane are very similar except for an overall multiplicative factor, which comes from the difference in impact distribution on the TCTs, shown in Fig. 7. The large fluctuations seen at large energies in some cases are caused by limited statistics.

The distributions are very similar also radially up to $r=3 \mathrm{~m}$ as shown for muons in Fig. 13, while differences at larger radii are caused by the different layout of the support tunnels in IR1 and IR5. Simulations show that if identical starting conditions are used in the IR1 and IR5 geometries, the results are nearly identical except at larger radii. Furthermore, the small difference in opening of the TCTs between 2010 and 2011 does not affect the shape of the distributions. 

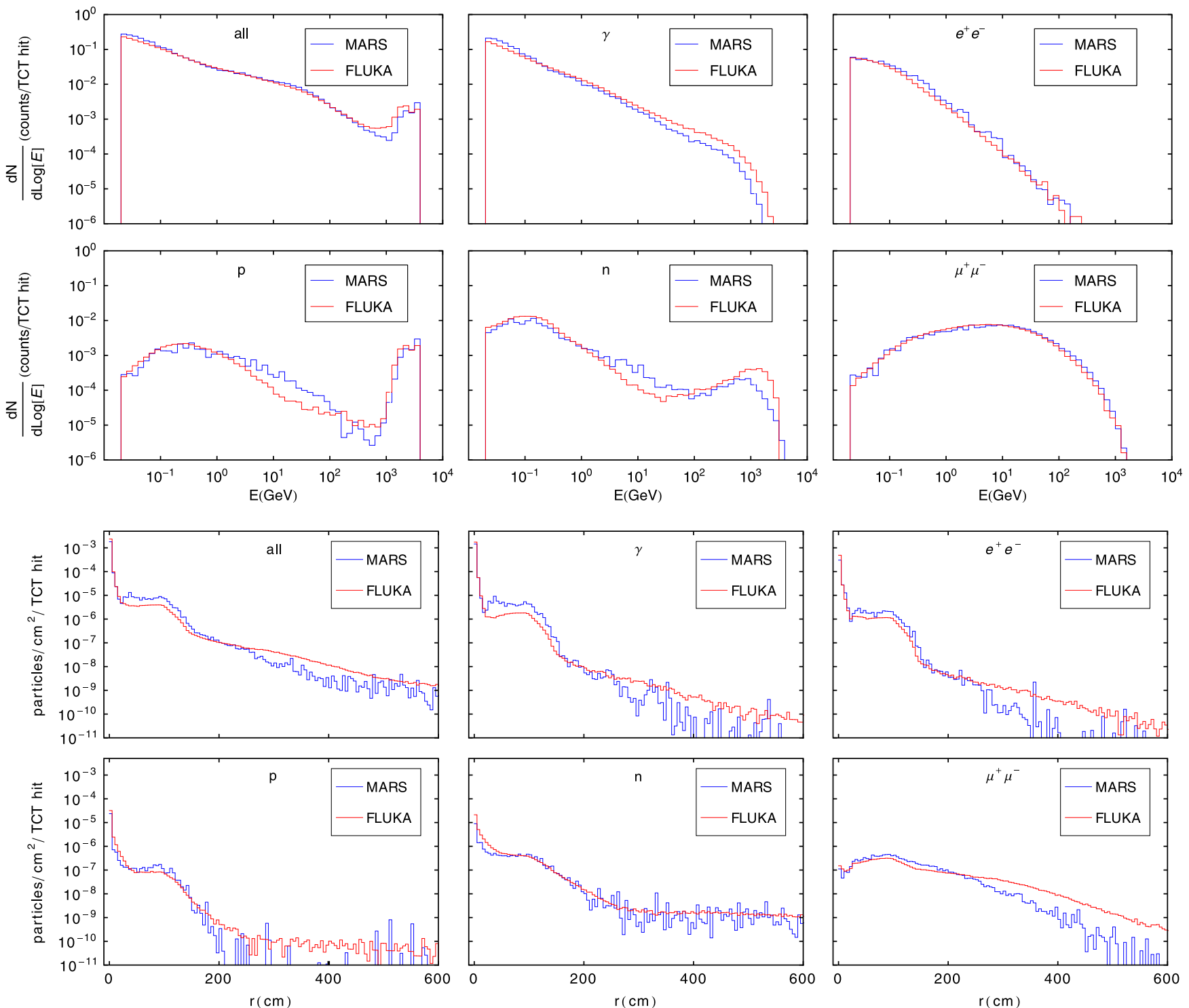

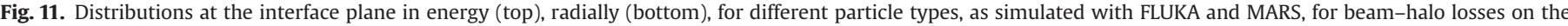
TCTs in IR5 B2 in the 2010 machine. The energy distributions are given as lethargy plots (normalized as $d N / d \log (E)=E d N / d E$ ).

The azimuthal distributions for IR1 are shown in Fig. 14. The muons are grouped in a two-spiked structure with peaks in the horizontal plane at the positive and negative sides of the $x$-axis. Both positive and negative muons are shown together in Fig. 14, but the two charges are deflected in opposite directions by the D1 bending magnet and therefore give rise to one spike each. This is discussed in more detail for beam-gas in Section 7.

In Fig. 15 we show the radial distribution for muons in IR1 with different energy cuts. The most energetic muons, with $E>100 \mathrm{GeV}$, give significant contributions at small radii but their distribution decays much more rapidly as a function of the radius than for lower energies.

\section{Simulations of beam-gas interactions around the ring}

Beam-gas interactions occur continuously around the ring during stored beam operation. The showers from very distant inelastic events do not reach the experimental detectors $[10,11]$ but protons scattered with a small variation in energy and angle can traverse long parts of the ring before they are lost. These particles, if lost close to the detector, contribute to the machineinduced background, as well as the showers from close-by inelastic beam-gas events.

We thus split the simulation of beam-gas in two parts: the events from local beam-gas, which occur close enough to the detector for some shower particles to reach the interface plane, and the global beam-gas scattering in all other parts of the ring. As in Ref. [11], we simulate the local beam-gas directly with FLUKA or MARS up to $z=550 \mathrm{~m}$ from the IP, tracking all shower particles, as described in Section 7, while for the global beam-gas, we apply a two-step approach similar to the simulations of beam-halo. First, SixTrack is used to track scattered protons around the ring. In a second step we can use FLUKA or MARS to simulate the showers of protons lost on the TCTs close to IP1 and IP5.

\subsection{SixTrack simulation of global beam-gas interactions}

We simulate elastic and inelastic scattering (including diffractive events) with FLUKA and tabulate the final state protons, which are passed to the SixTrack transport. The assumed cross-sections for the different gas elements are presented in Table 2. We track 

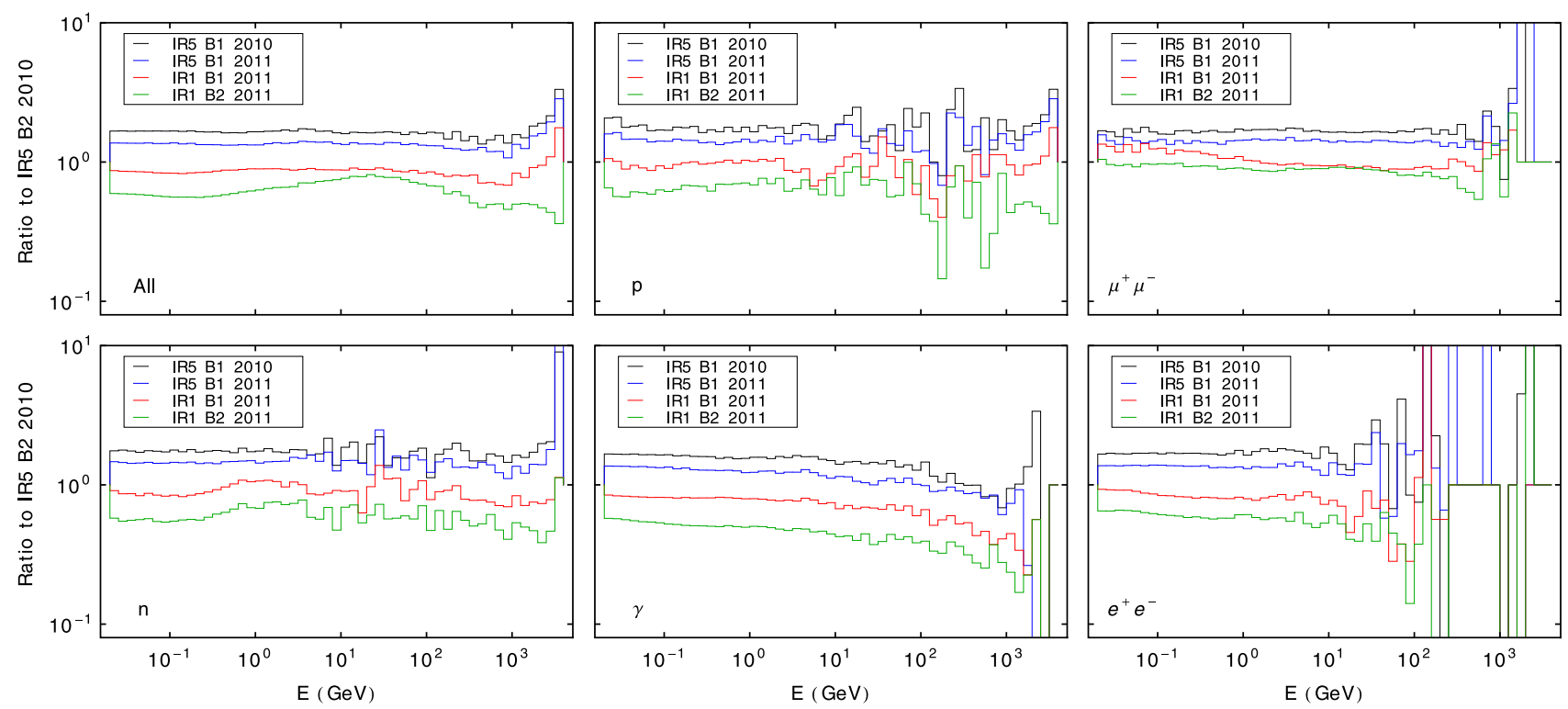

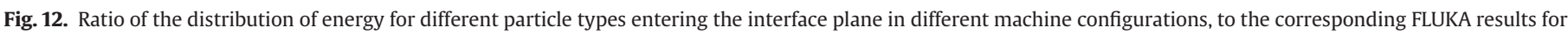

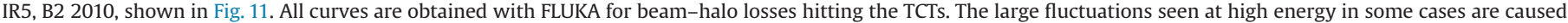
by limited statistics.

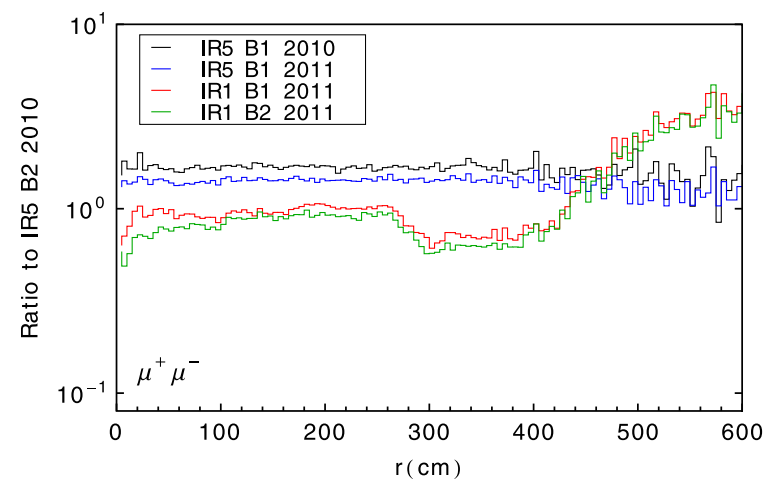

Fig. 13. Ratio of the radial distribution of muons at the interface plane to the corresponding FLUKA distribution for IR5, B2 2010, shown in Fig. 11. The curves are obtained with FLUKA for beam-halo losses on the TCTs.

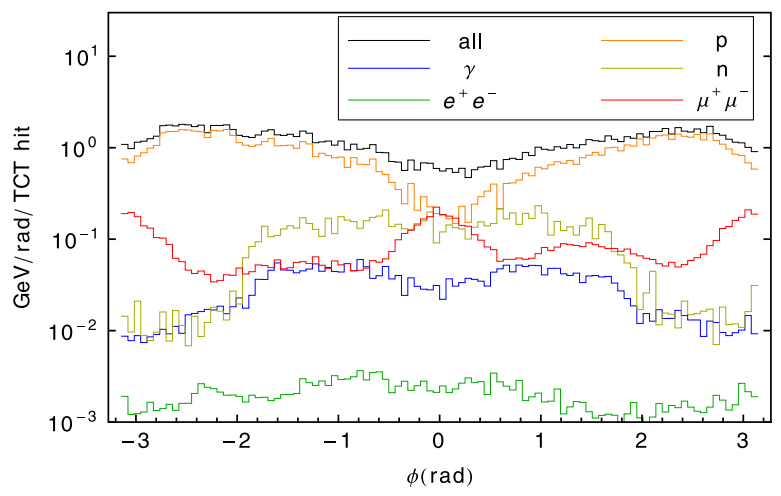

Fig. 14. Azimuthal distribution at the interface plane for different particle types, as simulated with FLUKA for beam-halo losses impinging on the TCTs in IR1 B1 in the 2011 machine, and weighted by the particle energy. The angle is defined as $\phi=0$ on the positive $x$-axis pointing towards the outside of the ring, and $\phi=\pi / 2$ at the positive $y$-axis.

only protons with an energy $E>2.8 \mathrm{TeV}$, since particles with lower energy are lost very close to the initial interaction due to the dispersion of the LHC lattice.

We then use SixTrack to track a bunch with a Gaussian transverse distribution around the LHC and sample beam-gas

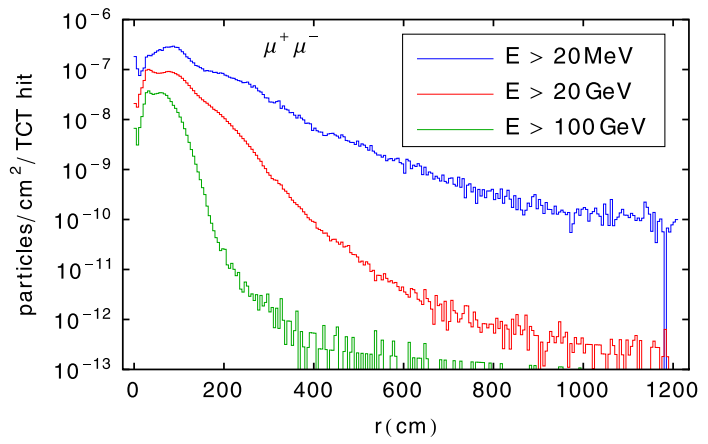

Fig. 15. Radial distribution of muons, as simulated with FLUKA for beam-halo losses impinging on the TCTs in IR1 B1 in the 2010 machine, with different energy cuts.

events from the pre-tabulated file with probabilities according to the local pressure. Only the 2011 machine, with the simulated pressure profile presented in Fig. 5, is simulated. We perform simulations for the three gas elements $(\mathrm{H}, \mathrm{C}, \mathrm{O})$ and for elastic and inelastic interactions and we consider both beams separately. No inelastic events were simulated in the region up to $550 \mathrm{~m}$ upstream of ATLAS and CMS, as these events are considered as local beam-gas and are simulated separately directly with FLUKA or MARS, as described in Section 7.

In each run the number of primary events is weighted according to the interaction rate given by Eq. (4). In this way, the obtained losses from all simulations can be combined in a straight-forward manner in the post-processing. The fraction of events in each simulation, weighted using the beam-gas crosssections in Table 2 and the gas densities in Fig. 5 are shown in Table 5. The total number of simulated events, summed over all simulations, is $1.88 \times 10^{7}$.

The local loss rate $N_{l}^{\prime}$ on a collimator or the machine aperture is obtained by scaling the number of simulated local losses $m$ by the ratio of the total loss rate in the ring $N^{\prime}$, given by Eq. (4) and Table 3, and the total number of simulated losses $M$

$N_{l}^{\prime}=m \frac{N^{\prime}}{M}$ 
Fig. 16 shows the simulated loss rates around the ring on the aperture of cold and warm machine elements, as well as on collimators, for elastic interactions on $\mathrm{H}$ and $\mathrm{O}$ in B1. Protons with large scattering angles are lost close to the initial interaction, while protons with smaller angles can travel a long distance, or even make several turns, before hitting collimators in other IRs. The loss distribution is different for interactions with $\mathrm{H}$ (12\% of all scattered protons lost on the cold aperture, $24 \%$ on TCTs) and O ( $2 \%$ lost on cold aperture, $12 \%$ on TCTs). The larger fraction of losses outside the cleaning insertion after scattering on $\mathrm{H}$ is caused by the larger scattering angles. In absolute numbers, protons interacting with $\mathrm{O}$ dominate losses both on the aperture and on collimators because of the larger rate (see Table 5). The remaining protons are lost on other collimators.

In Fig. 17 we show the total losses around the ring, summed over all gas species, for elastic and inelastic interactions. Inelastic interactions cause larger scattering angles or an immediate
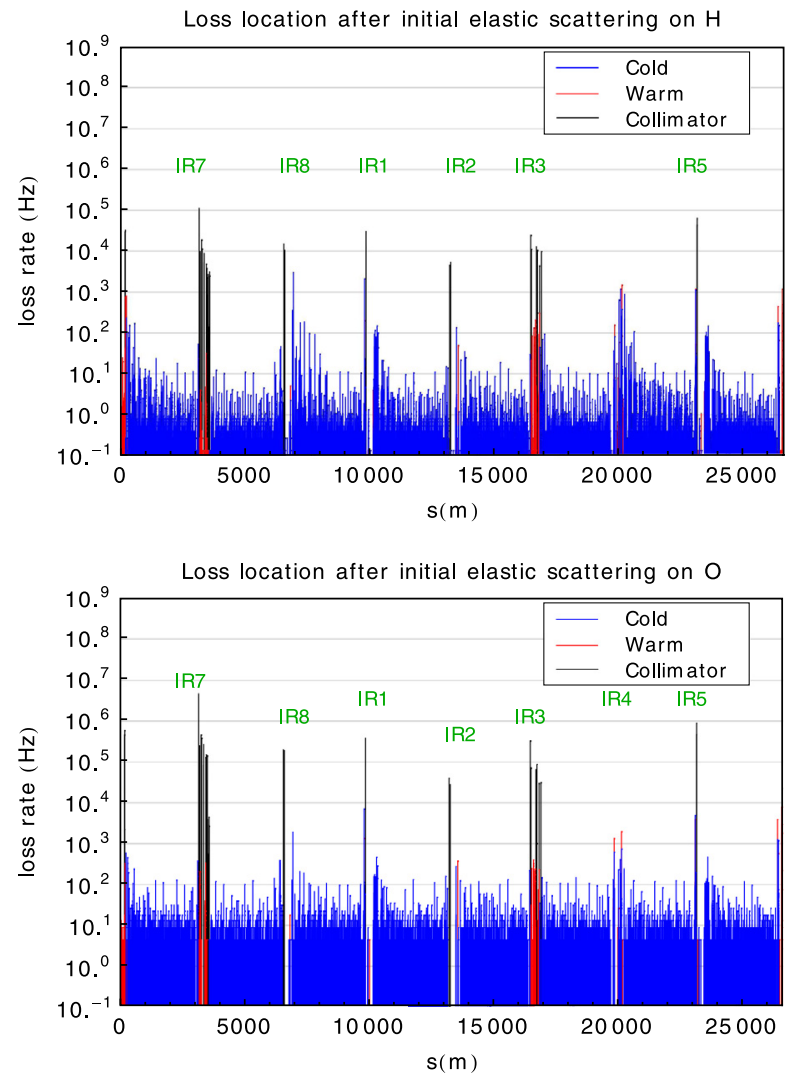

Fig. 16. Loss rates of beam protons in B1, simulated with SixTrack for fill 2028 (2011 machine), following elastic beam-gas interactions on $\mathrm{H}$ (top) and on $\mathrm{O}$ (bottom), which are pre-tabulated with FLUKA. We show the final loss location, where the scattered proton either undergoes an inelastic interaction in a collimator or hits the machine aperture, which for small scattering angles is often several machine octants downstream of the initial event. The loss rates shown in Table 3 and the loss fractions in Table 5 were used for normalization.

Table 5

Fraction of scattering events in percent per turn occurring on different gas species for inelastic and elastic interactions for the 2011 machine at 3.5 TeV. The weighting is done using Eq. (4) with the gas densities given by Fig. 5 and the interaction crosssections from Table 2.

\begin{tabular}{llrc}
\hline$\%$ & $\mathrm{H}$ & $\mathrm{C}$ & $\mathrm{O}$ \\
\hline Elastic & 0.6 & 9.2 & 20.0 \\
Inelastic & 2.6 & 22.3 & 45.3 \\
\hline
\end{tabular}

disintegration of the beam protons and produce a much higher rate of local losses close to the initial beam-gas interaction. Protons undergoing elastic or diffractive interactions have smaller deviations in energy and angle and therefore stay longer inside the ring and dominate the losses on collimators. It should be noted that the local rates on cold elements in IR1 and IR5 are lower since no inelastic interactions are simulated closer than $550 \mathrm{~m}$ upstream of these experiments.

The resulting loss rates on the TCTs in IR1 and IR5 are shown in Table 6. The loss rates on the TCTs in IR1 B1 are about half of the rates of B2, while the opposite holds true for IR5. To understand this, we study the origin of the particles impacting on the TCTs-an example is shown in Fig. 18. As can be seen, the betatron collimation in IR7 is very efficient in intercepting the protons from upstream beam-gas interactions, which is consistent with the leakage to the TCTs from IR7 in the beam-halo simulations (see Section 5.1). The momentum cleaning in IR3 intercepts a smaller fraction of the incoming particles because these collimators are placed at higher normalized amplitudes (see Table 1). Therefore, only two octants contribute to the rates on the IR1
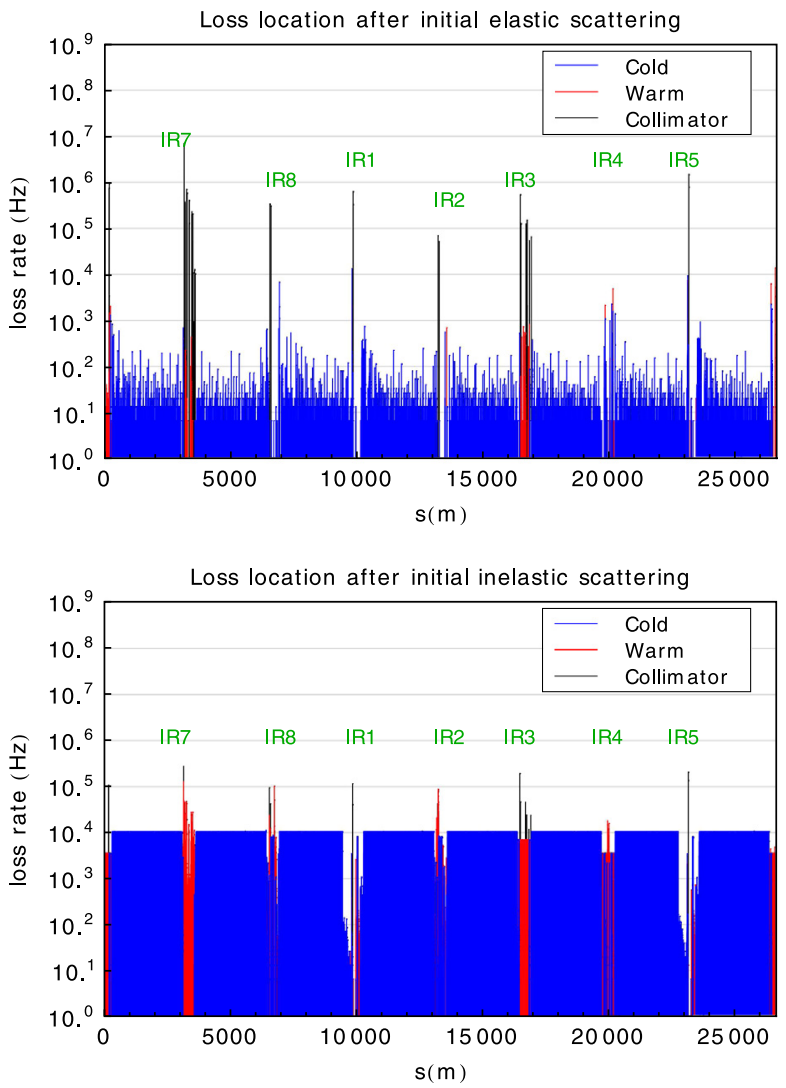

Fig. 17. Loss rates of beam protons in B1, simulated with SixTrack for fill 2028 (2011 machine), following elastic beam-gas interactions (top), and inelastic including diffractive beam-gas interactions (bottom) simulated with FLUKA, summed over all gas species. As in Fig. 16 we show the final loss location. The normalization is done as in Fig. 16.

\section{Table 6}

Loss rates on the TCTs, simulated with SixTrack, for global beam-gas. The results are normalized using data for fill 2028 from 2011 and are summed over the horizontal and vertical TCTs. Our results are of the same order as the rates simulated for $7 \mathrm{TeV}$ in Ref. [36].

\begin{tabular}{lcc}
\hline$(\mathrm{MHz})$ & IR1 & IR5 \\
\hline B1 & 1.1 & 2.6 \\
B2 & 2.4 & 1.3 \\
\hline
\end{tabular}



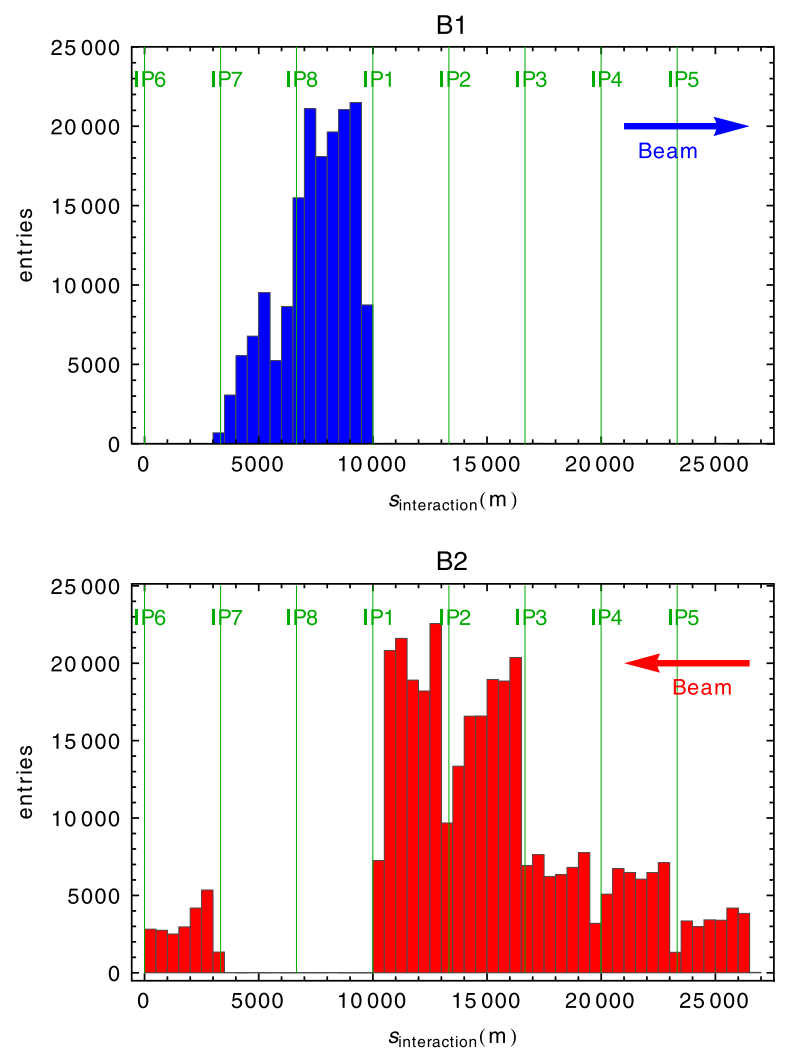

Fig. 18. Histograms of the $s$-location around the ring of the original beam-gas interaction for all protons hitting the TCTs in IR1 in B1 (top) and B2 (bottom). The arrows in the upper right corner indicate the beam direction. For better readability we have chosen $s=0$ in IR6.

TCTs in B1, while six octants contribute in B2, consistent with the observed asymmetry.

It should be noted that the momentum collimation in IR3 blocks about half of the incoming scattered particles and that tighter collimator gaps in IR3 could be beneficial for the loss rates on the TCTs. The TCTs in IR8 are also intercepting a substantial fraction of the incoming flux of scattered particles, while the TCTs in IR2 block much less, as these collimators are more open due to different optics at the interaction points [35].

\subsection{Shower simulation of global beam-gas interactions}

For the shower simulation, we start as for beam-halo with the position of inelastic interactions in the TCTs that are predicted by SixTrack. We neglect the small hit rate on the nearby machine aperture in the experimental IRs due to global beam-gas, since Fig. 17 shows that this is many orders of magnitude lower than the hit rate on the TCTs. We treat local inelastic beam-gas separately in Section 7.

The shower simulation of global beam-gas is thus very similar to the beam-halo case, apart from the starting positions, shown in Fig. 19, which are transversely deeper inside the jaws. Some energy distributions at the interface plane are shown in Fig. 20, where they are compared to the results from beam-halo and the radial distribution of muons is shown in Fig. 21. The results have been normalized to the reference case presented in Fig. 11 for easier comparisons. As can be seen, the distributions are very similar, apart from a global scale factor which depends on the starting distribution of interactions in the TCTs. The leakage to the interface plane per proton lost on the TCTs is smaller by about a factor 3 for global beam-gas than for halo, since the deeper impacts cause more of the shower to be contained within the tungsten jaws. As for beam-halo, some differences between IR1 and IR5 are seen at $r>300 \mathrm{~cm}$, caused by the different layout of the support tunnels.

It should also be noted that, even more than for beam-halo, there is a high resemblance between the distributions at IR1 and IR5, both at the TCTs and at the interface plane. This can be understood from the fact that the interaction rates in the upstream arcs are assumed similar, meaning that the main difference comes from the geometry and the asymmetry of the location of the cleaning insertions in the ring. ${ }^{7}$

\section{Simulations of beam-gas events close to the experiments}

For the local beam-gas simulations, we use the same geometries as for the beam-halo shower simulations and consider inelastic events occurring upstream of the interface plane on the incoming beam orbit over the whole geometry model. As we assume the same pressure profile for the two incoming beams, only one simulation is performed per IR. It should be noted that the FLUKA geometry used in this work for IR5 ends at $z=150 \mathrm{~m}$, while for IR 1 it extends to $z=550 \mathrm{~m}$. The effect of extending the simulated region in IR 1 from $z=150 \mathrm{~m}$ to $z=550 \mathrm{~m}$ gives an increase of the particle fluxes at the interface plane by up to 20 $\%$, depending on particle type and energy, and we thus expect a similar increase in IR5 if a larger part of the geometry would be considered also there.

Inelastic interactions of $3.5 \mathrm{TeV}$ protons with gas nuclei are sampled along the beam trajectory in FLUKA. A uniform probability distribution in the longitudinal coordinate along the ideal orbit is used, which is equivalent to an underlying assumption of a constant pressure profile. This does evidently not correspond to reality. However, in the output files all correlations are kept between the secondaries reaching the interface plane and the initial interaction. Therefore, particles from an arbitrary pressure profile can be reproduced by simple post-processing routines: single events with all resulting secondaries can be sampled with a probability given by the local pressure at the position of the initial interaction.

For the elastic interactions treated in Section 6, the nucleus type interacting with the beam protons is important, since the scattering angle and energy deviation can vary significantly between nuclei. This influences where in the ring losses on the machine aperture or collimators occur. Inelastic interactions, on the other hand, involve nucleon-nucleon reactions and their final state has a only minor dependence on the target nucleus, within a certain mass range. For simplicity and generality, we therefore sample events on nitrogen, which has atomic and mass numbers between $\mathrm{C}$ and $\mathrm{O}$. This is a good approximation for $\mathrm{C}$ and $\mathrm{O}$, with a potential uncertainty for $\mathrm{H}$. However, even though the $\mathrm{H}$ density is high close to the IP, the total number of interactions is more than an order of magnitude higher on $\mathrm{C}$ and $\mathrm{O}$ with higher crosssections, which justifies our approximation. With this approach, combined with the uniform sampling, the lengthy FLUKA simulation is completely independent on the pressure profile, which is instead used in a second step to re-normalize the results. A single FLUKA simulation can therefore be used with any pressure profile.

For the normalization, we use the pressure profile in Fig. 5. The total event rate between the interface plane and $s=550 \mathrm{~m}$ is given by Eq. (4) if we take the integral only over this distance instead of the whole ring and sum over $j$. We replace the integral by a sum over the discrete longitudinal bins, where we make the integration bins small enough to have a constant gas density. If $M_{k}$ primary

\footnotetext{
${ }^{7}$ Because of the layout, IR1 B1 is expected to be similar to IR5 B2 and vice versa as in Table 6.
} 

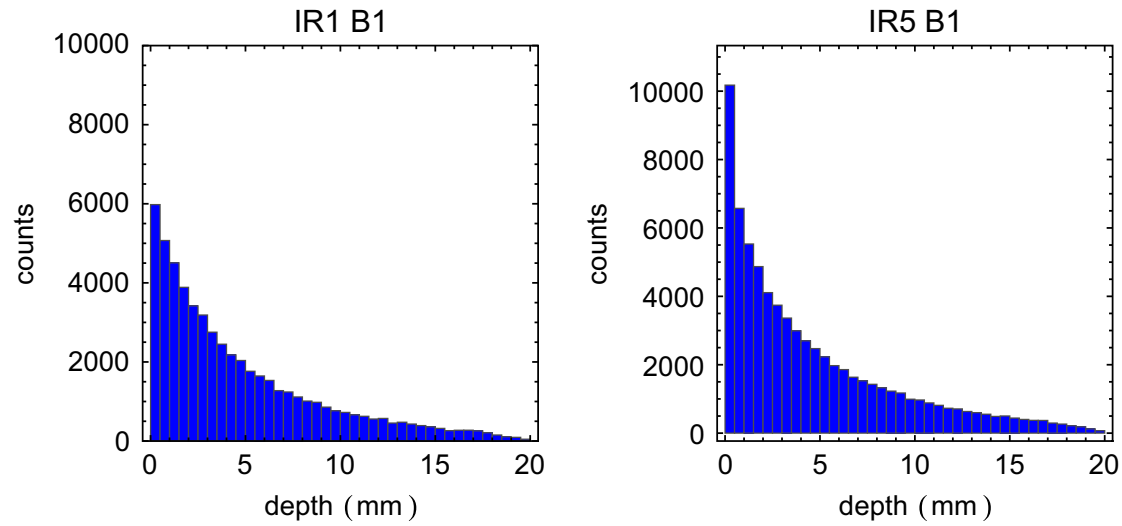

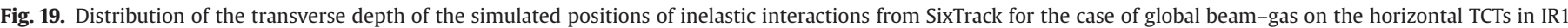
and IR5 for B1.
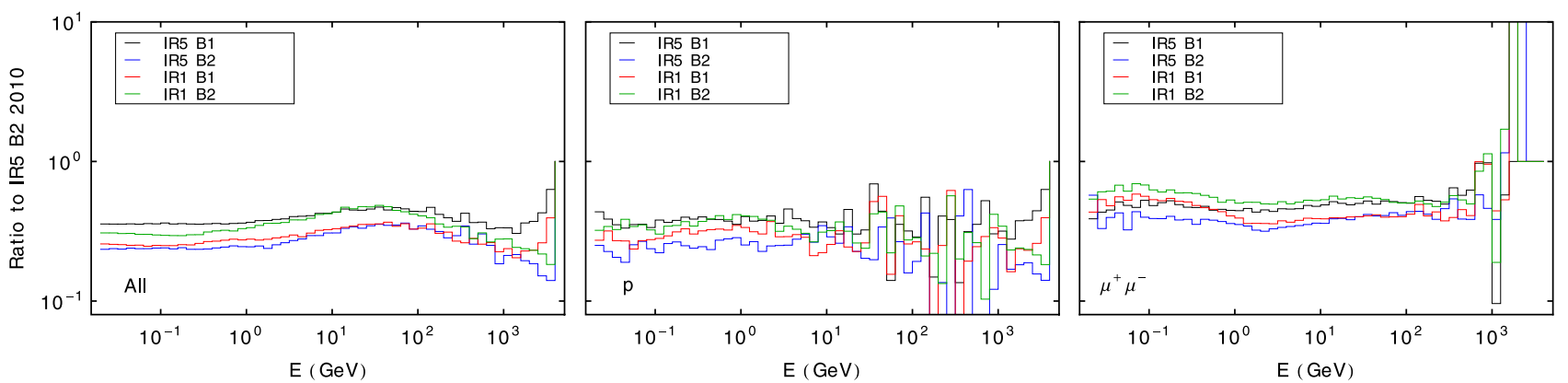

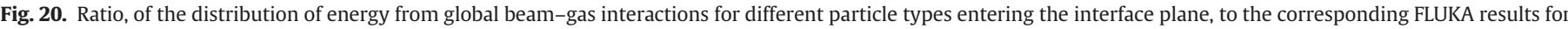

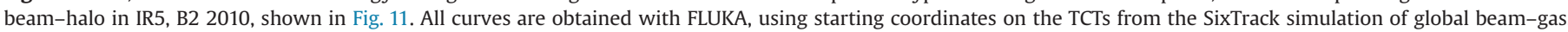
The large fluctuations at high energy are caused by limited statistics.

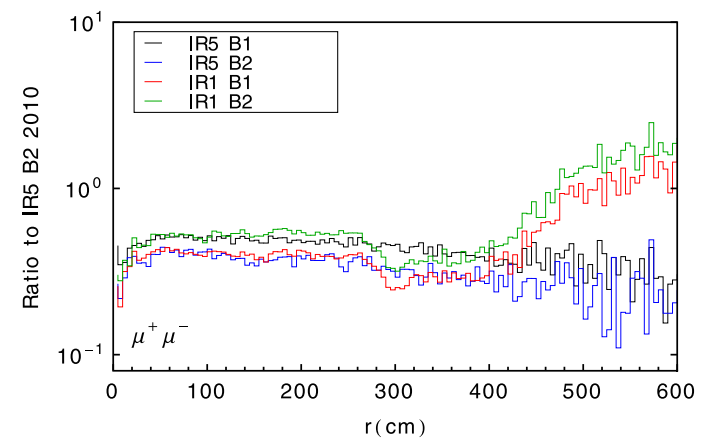

Fig. 21. Ratio of the radial distribution for global beam-gas of muons entering the interface plane per proton lost on the TCTs, to the corresponding results for beamhalo in IR5 B2 2010, shown in Fig. 11. The curves are obtained with FLUKA.

events are simulated in bin $k$ and they produce $m_{k}$ particles at the interface plane, the total rate $n^{\prime}$ at the interface plane is

$n_{\mathrm{gas}}^{\prime}=\sum_{k} \frac{N}{T_{\mathrm{rev}} M_{k}} \sigma \rho_{k} \Delta s_{k} m_{k}$

Here $\Delta s_{k}$ and $\rho_{k}$ are the length of bin $j$ and the nitrogen-equivalent gas density in it. ${ }^{8}$ Furthermore, $\sigma$ is the nitrogen cross-section. Both $M_{k}$ and $\Delta s_{k}$ are constant if the bins are chosen with equal length. If several gas species would be simulated instead of only nitrogen, Eq. (6) should be summed over them.

\footnotetext{
${ }^{8}$ This is calculated by weighting the densities of all gas species by the ratio of the respective cross-sections to the nitrogen cross-section and summing all contributions.
}

In Fig. 22 we show some simulated distributions at the interface plane at IR1, normalized to rates. They are qualitatively similar to the case with the shower starting at the TCTs but have a much stronger peak in the center of the beam pipe, as there is no collimator blocking the initial shower. The results are also qualitatively similar to the MARS simulations in Refs. $[10,11]$ for the $7 \mathrm{TeV}$ nominal LHC.

The distribution in distance $z$ from IP1 of the initial beam-gas interaction creating the muons at the IR1 interface plane is shown in Fig. 23. The contribution of muons at the interface plane from the inner triplets $(22 \mathrm{~m} \lesssim z \lesssim 55 \mathrm{~m}$ ) is high and then decreases gradually over the length of the first separation dipole between $60 \mathrm{~m}$ and $85 \mathrm{~m}$. The TCTs at $z=145 \mathrm{~m}$ have a local pressure spike (Fig. 5) but block efficiently large parts of the upstream events, although the contributions from the matching section quadrupoles ( $z \simeq 190 \mathrm{~m}, z \simeq 220 \mathrm{~m}$, and $z \simeq 265 \mathrm{~m}$ ) is clearly visible. Upstream of $z=270 \mathrm{~m}$, the bending magnets of the arc act as a spectrometer. Most of the particles arriving at the interface plane from events beyond this point are protons with small energy deviations, which are not filtered out by the bending. Nevertheless some muons from this region make it to the detectors at large radii.

In Fig. 24 we show the azimuthal distribution of high-energy muons $(E>20 \mathrm{GeV})$ in IR1, separated by charge, for different $z$ positions of the initial beam-gas event. Clear structures can be observed: the muons created closer to the IP than $53 \mathrm{~m}$ pass only through the inner triplet quadrupoles, which concentrate them in the mid-planes. The effect is similar for the two charges, although positively charged muons are found in larger amounts in the horizontal plane and negative muons in the vertical, due to the opposite focusing and defocusing. On the other hand, muons created farther away traverse the D1 dipole field, which separates 



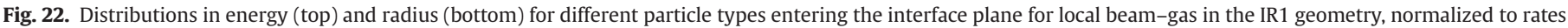


distributions are given as lethargy plots as in Fig. 11.

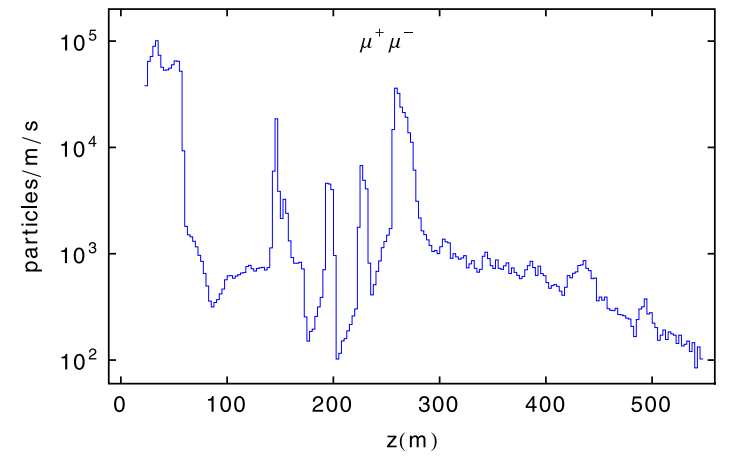

Fig. 23. Distribution of muons at the interface plane, binned in longitudinal coordinate $z$ (from IP1) of the initial beam-gas interaction that produced the muons. The curves are obtained with FLUKA and normalized to rates using the pressure profile of Fig. 5.

the two charges in the horizontal plane. This effect is stronger than the effect of the quadrupoles. This latter distribution is seen also in the simulations starting at the TCT, where all muons pass the D1.
It should be noted that muons created upstream of $z=153 \mathrm{~m}$ pass first the D2 dipole, which bends in the opposite direction, meaning that the muon charges are eventually mixed.

\section{Quantitative comparison of particle rates from beam-halo and beam-gas}

Using the simulations and machine conditions for fill 2028, described in previous sections, we make a quantitative comparison between beam-halo and beam-gas. For local beam-gas, the normalization is given by Eq. (6) as explained in Section 7. The shower simulations of global beam-gas and beam-halo are normalized to the respective hit rates on the TCTs. For global beam-gas these rates are shown in Table 6 .

For beam-halo, we consider the estimated loss rate on the TCPs of $570 \mathrm{MHz}$, shown in Table 3, and multiply by the leakage between primary and tertiary collimators as simulated with SixTrack (Table 4). The resulting loss rates on the TCTs from beamhalo are well below $100 \mathrm{kHz}$, while the loss rates from global beam-gas are above $1 \mathrm{MHz}$. This more than compensates for the 
smaller leakage of particles to the interface plane per proton on the TCT (see Section 6.2).

Figs. 25 and 26 show the obtained rates of muons and all particles at the interface plane at IR1, binned in energy or radius, at the beginning of fill 2028. It should be noted that for local beamgas, the same simulation has been used for both beams. It can be seen that the total beam-gas contribution at the interface plane is in all cases at least one order of magnitude higher than the beamhalo contribution. For muons of $10-100 \mathrm{GeV}$, at $r \gtrsim 150 \mathrm{~cm}$, global beam-gas gives comparable or even larger contributions than local beam-gas.

In Table 7 we show the total rates of muons with an energy above $100 \mathrm{GeV}$, which are entering the interface plane outside the beam pipe $(r>2.5 \mathrm{~cm})$, for both IRs and beams. These particles are most important in terms of the risk of causing fake jets. As can be seen, local or global beam-gas give the largest contributions depending on IR and beam, with rates in the range between $480 \mathrm{~Hz}$ and $1.4 \mathrm{kHz}$. We remind that the results for local beam-gas in IR5 are likely to be underestimated by about $20 \%$ because of the shorter geometry as explained in Section 7 . The rates from beamhalo are instead between $9 \mathrm{~Hz}$ and $54 \mathrm{~Hz}$.

It should also be noted that there is an asymmetry between B1 and B2 coming from global beam-gas, due to the difference in rate on the TCTs found in the SixTrack simulations (see e.g. Fig. 18). In both IR1 and IR5, the muon rates are expected to be 1.6-1.7 times higher on the side of the experiment that is farther away from IR7. This is compatible with the observed differences in ATLAS [30],

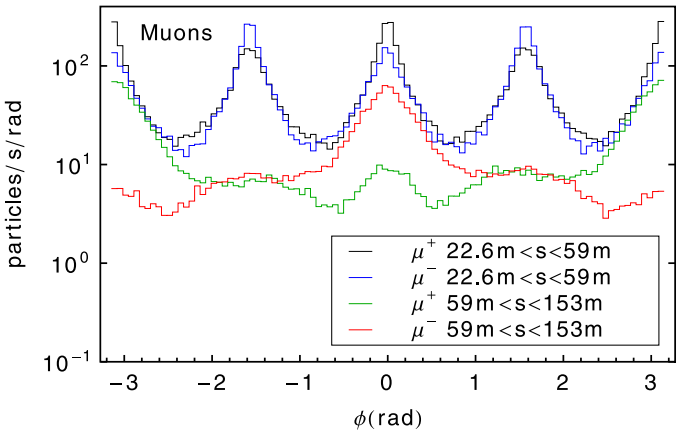

Fig. 24. Angular distribution of muons with $E>20 \mathrm{GeV}$, simulated with FLUKA for local beam-gas, separated by charge and initial longitudinal coordinate $z$ from the IP of the position of the initial beam-gas interaction. where the registered muon rate is shown to be a factor 1.75 higher in $\mathrm{B} 2$.

\section{Cross-talk between experiments}

Another source of background, with characteristics similar to those discussed before, is the cross-talk between different IPs, when particles scattered in the collisions in one experiment travel through the ring and hit the TCTs in front of another experiment. This is treated for the nominal $7 \mathrm{TeV}$ configuration in Ref. [36] for IP2 and IP8. The hit rates on the TCTs in these IRs are estimated to be a fraction $10^{-7}-10^{-4}$ of the collision rate at the other IPs. With the luminosities observed in 2010-2011, this would imply hit rates on the TCTs of the order of a few to tens of $\mathrm{kHz}$, which is comparable to the rates simulated for beam-halo.

It should be noted that cross-talk is likely to be more important for IR1 B2 and IR5 B1 due to the location of the IR7 cleaning insertion which, in analogy to the global beam-gas, is expected to intercept a large fraction of the scattered protons. The cross-talk depends also on the betatron phase-advance conditions, which in the horizontal plane is not far from $\pi / 2$ between IR1 and the IR5 TCTs. Therefore, this cross-talk risks to be more important than the cross-talk with the low-luminosity experiments. In particular, for other LHC configurations with significantly higher luminosity than in 2011, this should be studied in detail. To quantitatively assess the importance of cross-talk in IR1 and IR5, new simulations are needed, accounting for the betatron phase advance between the IPs as well as the angle-energy distribution of the relevant collision products. This is left as future work.

\section{Conclusions}

We simulate particle fluxes entering the ATLAS and CMS experiments from the LHC machine, causing experimental background, using SixTrack, FLUKA, and MARS. The simulations stop at a defined interface plane between the machine and the detector, located at $22.6 \mathrm{~m}$ from the collision point. Two background sources are considered-halo protons hitting the tertiary collimators (TCTs), driving a shower of which a small part reaches the detectors, and interactions between beam protons and the residual gas. The latter source is split in a global contribution, where the


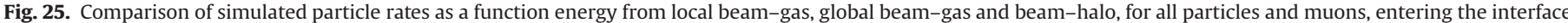
plane in IR1 as simulated with FLUKA for the 2011 machine configuration. The energy distributions are given as lethargy plots as in Fig. 11. 

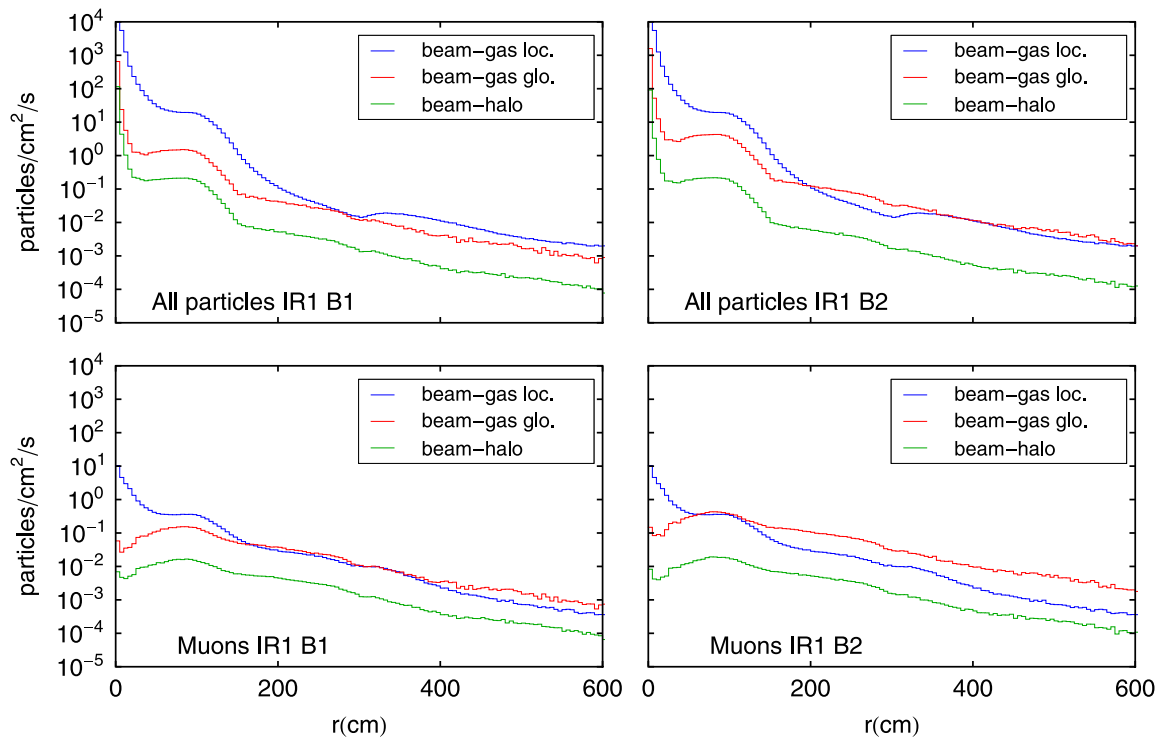

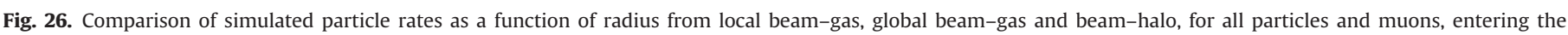
interface plane in IR1 as simulated with FLUKA for the 2011 machine configuration.

Table 7

Simulated total rates of muons from different background sources, with $E>100 \mathrm{GeV}$ and outside the central beam pipe $(r>2.5 \mathrm{~cm})$, entering the interface plane in IR1 and IR5 in the 2011 LHC configuration. It should be noted that the simulation for local beam-gas in IR5 ends at $z=150 \mathrm{~m}$, while it ends at $z=550 \mathrm{~m}$ in IR1. This is likely to cause an underestimation of the local beam-gas rate in IR5 by about $20 \%$. The values in brackets for local beam-gas in B1 were assumed the same as in B2, as no separate simulation was performed for B1 because of the identical optics, FLUKA geometry and pressure profile.

\begin{tabular}{lcclc}
\hline IR and beadm & Beam-halo rate $(\mathrm{Hz})$ & Global beam-gas rate $(\mathrm{Hz})$ & Local beam-gas rate $(\mathrm{Hz})$ & Total rate $(\mathrm{Hz})$ \\
\hline IR1 B1 & 49 & 480 & $(740)$ & 1242 \\
IR1 B2 & 54 & 1220 & 740 & 1987 \\
IR5 B1 & 38 & 1380 & $(670)$ & 2066 \\
IR5 B2 & 9 & 560 & 670 & 1234 \\
\hline
\end{tabular}

initial scattering event occurs far from the detector, and a local contribution from close-by events. We consider the $3.5 \mathrm{TeV}$ proton beams used in 2010 and 2011.

In order to normalize the simulation results to rates, logged LHC data are used for a quantitative analysis of different contributions to the total loss rate from the LHC beams. In the 2011 run, the beam losses were dominated by halo particles hitting collimators and inelastic proton collisions in the experiments. The first contribution was equally or more important than the latter. We estimate that beam-gas scattering caused about $10 \%-20 \%$ of the total losses.

For protons hitting the TCTs, the particle leakage to the interface plane per TCT hit is similar in all cases, regardless of the experiment and the source (global beam-gas or beam-halo), apart from a multiplicative factor, which depends on the difference in impact distribution on the TCTs. The average fraction of the initial proton energy reaching the interface plane is in all studied cases of the order of a permil and about a factor 3 higher for beam-halo than for global beam-gas, where the TCT hits are deeper.

For local beam-gas, events in the straight section closest to the detector, in particular in the inner triplet, are most important in terms of amount of energy reaching the interface plane, but at large radii also muons created farther away, even in the arc, contribute.

The comparison between FLUKA and MARS of the distributions obtained at the interface plane, using identical source terms, shows a very good agreement. This increases our confidence in the results, since both the codes themselves as well as the geometry models are implemented independently. It serves also an evidence of the excellent consistency between the two codes.
In absolute numbers, global and local beam-gas events are the dominating sources of the particle rates at the interface plane during a typical 2011 fill. Beam-halo is found to be at least one order of magnitude less important. Our simulations show an asymmetry of a factor $1.6-1.7$ between the muon rates at the two sides of the experiments, which comes from the asymmetry in the distance along the incoming beam orbit to the cleaning insertion IR7. The predicted factor is compatible with ATLAS measurements [30].

Our results can be used as a starting point for a further simulation of the signals caused by the particles entering the experimental detectors from the machine.

\section{Acknowledgments}

We would like to thank P. Hagen for providing the magnetic field map of the D1 dipole. We are also grateful to N. Bacchetta, W. Bell, P. Bhat, L. Esposito, S. Gibson, M. Guthoff, W. Kozanecki, G. Lanza, S. Redaelli, A. Rossi, and E. Skordis for helpful discussions and input.

\section{References}

[1] O.S. Brüning, P. Collier, P. Lebrun, S. Myers, R. Ostojic, J. Poole, P. Proudlock (Eds.), LHC Design Report v.1: The LHC Main Ring, CERN-2004-003-V1.

[2] L. Evans, P. Bryant (Eds.), Journal of Instrumentation (3) (2008) S08001.

[3] A.I. Drozhdin, M. Huhtinen, N.V. Mokhov, Nuclear Instruments and Methods in Physics Research Section A 381 (2-3) (1996) 531. 
[4] K.M. Potter (Ed.), Proceedings of the Workshop on LHC Backgrounds, CERN, Geneva, March 22, 1996, CERN-2009-003.

[5] N. Mokhov, Nuclear Physics B - Proceedings Supplements 51 (1) (1996) 210.

[6] I. Azhgirey, I. Baishev, V. Talanov, K. Potter, Machine induced background in the high luminosity experimental insertion of the LHC project, in: Proceedings of the European Particle Accelerator Conference 2004, Lucerne, Switzerland, 2004, p. 755

[7] V. Talanov, Estimation of the machine induced background for the commissioning period with tertiary collimators in the IR1 of the LHC, LHC Project Note 371, CERN.

[8] G. Corti, V. Talanov, Aspects of machine induced background in the LHC Experiments, LHC Project Workshop - Chamonix XV, 2006, p. 178.

[9] R. Alemany-Fernandez, H. Burkhardt, M. Ferro-Luzzi, M. Lamont, A. Macpherson, S. Redaelli (Eds.), Proceedings of the LHC Workshop on Experimental Conditions and Beam-Induced Detector Backgrounds, CERN, Geneva, Switzerland, April 2008, CERN-2009-003.

[10] N.V. Mokhov, T. Weiler, Machine-induced backgrounds: their origin and loads on ATLAS/CMS, Fermilab-Conf-08-147-APC; ibid pp. 37-43.

[11] A. Drozhdin, N. Mokhov, S. Striganov, Beam losses and background loads on collider detectors due to beam-gas interactions in the LHC, in: Proceedings of the 2009 Particle Accelerator Conference, Vancouver, Canada, 2009, pp. 25492551.

[12] ATLAS Collaboration, Journal of Instrumentation (3) (2008) S08003.

[13] CMS Collaboration, Journal of Instrumentation (3) (2008) S08004.

[14] R.W. Assmann, Collimators and Beam Absorbers for Cleaning and Machine Protection, LHC Project Workshop - Chamonix XIV, 2005, p. 261.

[15] R.W. Assmann, et al., The final collimation system for the LHC, in: Proceedings of the European Particle Accelerator Conference 2006, Edinburgh, Scotland, 2006, p. 986.

[16] D. Wollmann, et al., First cleaning with LHC collimators, in: Proceedings of the International Particle Accelerator Conference 2010, Kyoto, Japan, 2010, p. 1237.

[17] J.B. Jeanneret, Physical Review Special Topics - Accelerators and Beams 1 (8) (1998) 081001

[18] F. Schmidt, SixTrack. Users Reference Manual, CERN/SL/94-56-AP.

[19] T. Trenkler, J. Jeanneret, K2, a software package evaluating collimation systems in circular colliders (manual), CERN SL/94105 (AP).

[20] G. Robert-Demolaize, R. Assmann, S. Redaelli, F. Schmidt, A new version of sixtrack with collimation and aperture interface, in: Proceedings of the Particle Accelerator Conference 2005, Knoxville, 2005, p. 4084.
[21] A. Ferrari, P. Sala, A. Fassò, J. Ranft, FLUKA: a multi-particle transport code, CERN Report CERN-2005-10.

[22] G. Battistoni, S. Muraro, P.R. Sala, F. Cerutti, A. Ferrari, S. Roesler, A. Fasso, J. Ranft, The FLUKA code: Description and benchmarking, Hadronic Shower Simulation Workshop 2006, Fermilab 6-8 September 2006, AIP Conference Proceeding 896, 2007, pp. 31-49.

[23] 〈http://www.fluka.org $\rangle$.

$24]$ R. Bruce, et al., Simulations and measurements of cleaning with $100 \mathrm{MJ}$ beams in the CERN Large Hadron Collider, in: Proceedings of the International Particle Accelerator Conference 2013, Shanghai, China.

[25] V. Vlachoudis, FLAIR: a powerful but user friendly graphical interface for FLUKA, in: Proceedings of the International Conference on Mathematics, Computational Methods and Reactor Physics (MC 2009), Saratoga Springs, New York. URL 〈http://www.fluka.org/flair/〉.

[26] N.V. Mokhov, The MARS code system user's guide, Fermilab-FN 628. URL $\langle$ http://www-ap.fnal.gov/MARS/〉.

27] N. Mokhov, S. Striganov, MARS15 Overview, Fermilab-Conf-07/008-AD (2007); in: Proceedings of Hadronic Shower Simulation Workshop, Fermilab, September 2006, AIP Conference Proceedings 896, pp. 50-60.

[28] A. Rossi, Vasco vacuum stability code: multi-gas code to calculate gas density, LHC Project Note 341, CERN.

[29] G. Bregliozzi, G. Lanza, V. Baglin, J. Jimenez, Vacuum 86 (11) (2012) 1682.

[30] ATLAS Collaboration, Journal of Instrumentation 8 (07) (2013) P07004, URL 〈http://stacks.iop.org/1748-0221/8/i=07/a=P07004〉.

[31] B. Salvachua, R. Bruce, M. Brugger, F. Cerutti, S. Redaelli, Estimate of warm magnets lifetime in the betatron and momentum cleaning insertions of the LHC, in: Proceedings of the International Particle Accelerator Conference 2013. Shanghai, China.

[32] TOTEM Collaboration, Europhysics Letters (2011) 21002.

33] G. Robert-Demolaize, Design and Performance Optimization of the LHC Collimation System, Ph.D. Thesis, Universite Joseph Fourier, Grenoble, 2006.

[34] C. Bracco, Commissioning Scenarios and Tests for the LHC Collimation System, Ph.D. Thesis, EPFL Lausanne, 2008.

[35] R. Bruce, R. Assmann, How low can we go? Getting below $\beta^{*}=3.5 \mathrm{~m}$, in: Proceedings of the 2010 LHC Beam Operation Workshop, Evian, France, 2010 p. 133.

[36] Y. Levinsen, Machine Induced Experimental Background Conditions in the LHC, Ph.D. Thesis, Oslo University, 2012. 